\title{
Genetics Responses to Hypoxia and Reoxygenation Stress in Larimichthys crocea Revealed via Transcriptome Analysis and Weighted Gene Co-Expression Network
}

\author{
Yibo Zhang ${ }^{1,2,+}$, Jie Ding ${ }^{1,2,+}$, Cheng Liu ${ }^{1,2}$, Shengyu Luo ${ }^{1}$, Xinming Gao ${ }^{1}$, Yuanjie Wu ${ }^{1}$, Jingqian Wang ${ }^{1}$, \\ Xuelei Wang ${ }^{2}$, Xiongfei $\mathrm{Wu}^{2}$, Weiliang Shen ${ }^{2, *}$ and Junquan $\mathrm{Zhu}{ }^{1, *}$ \\ 1 Key Laboratory of Applied Marine Biotechnology of Ministry of Education, College of Marine Sciences, \\ Ningbo University, 169 South Qixing Road, Ningbo 315832, China; zyb15058456761@163.com (Y.Z.); \\ dj13821799852@163.com (J.D.); 1801091036@nbu.edu.cn (C.L.); 1701091031@nbu.edu.cn (S.L.); \\ nbugxm4851@163.com (X.G.); 1911091104@nbu.edu.cn (Y.W.); 2001130069@nbu.edu.cn (J.W.) \\ 2 State Key Laboratory of Large Yellow Croaker Breeding, Ningbo Academy of Oceanology and Fishery, \\ Juxian Road, Ningbo 315103, China; xlwang126@163.com (X.W.); wxiongfei@hotmail.com (X.W.) \\ * Correspondence: sweleon@163.com (W.S.); zhujunquan@nbu.edu.cn (J.Z.); Tel.: +86-153-8137-7660 (W.S.); \\ +86-139-5784-1679 (J.Z.) \\ + These authors contributed equally to this work.
}

check for updates

Citation: Zhang, Y.; Ding, J.; Liu, C.; Luo, S.; Gao, X.; Wu, Y.; Wang, J.; Wang, X.; Wu, X.; Shen, W.; et al. Genetics Responses to Hypoxia and Reoxygenation Stress in Larimichthys crocea Revealed via Transcriptome Analysis and Weighted Gene Co-Expression Network. Animals 2021, 11, 3021. https://doi.org/ 10.3390/ani11113021

Academic Editors:

Athanasios Samaras and Arkadios Dimitroglou

Received: 3 September 2021 Accepted: 29 September 2021 Published: 20 October 2021

Publisher's Note: MDPI stays neutral with regard to jurisdictional claims in published maps and institutional affiliations.

Copyright: () 2021 by the authors Licensee MDPI, Basel, Switzerland. This article is an open access article distributed under the terms and conditions of the Creative Commons Attribution (CC BY) license (https:// creativecommons.org/licenses/by/ $4.0 /)$.
Simple Summary: Hypoxia, which occurs frequently in aquaculture, can cause serious harm to all aspects of the growth, reproduction and metabolism of cultured fish. Due to the intolerance of Larimichthys crocea to hypoxia, Larimichthys crocea often floats head or even dies under hypoxic environment. However, the molecular mechanism of hypoxia tolerance in Larimichthys crocea has not been fully described. Therefore, the aim of this study was to explore the hub regulatory genes under hypoxic stress environment by transcriptome analysis of three key tissues (liver, blood and gill) in Larimichthys crocea. We identified a number of important genes that exercise different regulatory functions. Overall, this study will provide important clues to the molecular mechanisms of hypoxia tolerance in Larimichthys crocea.

Abstract: The large yellow croaker (Larimichthys crocea) is an important marine economic fish in China; however, its intolerance to hypoxia causes widespread mortality. To understand the molecular mechanisms underlying hypoxia tolerance in L. crocea, the transcriptome gene expression profiling of three different tissues (blood, gills, and liver) of L. crocea exposed to hypoxia and reoxygenation stress were performed. In parallel, the gene relationships were investigated based on weighted gene co-expression network analysis (WGCNA). Accordingly, the Gene Ontology and Kyoto Encyclopedia of Genes and Genomes enrichment analysis showed that several pathways (e.g., energy metabolism, signal transduction, oxygen transport, and osmotic regulation) may be involved in the response of $L$. crocea to hypoxia and reoxygenation stress. In addition, also, four key modules (darkorange, magenta, saddlebrown, and darkolivegreen) that were highly relevant to the samples were identified by WGCNA. Furthermore, some hub genes within the association module, including RPS16, EDRF1, KCNK5, SNAT2, PFKL, GSK-3 $\beta$, and PIK3CD, were found. This is the first study to report the coexpression patterns of a gene network after hypoxia stress in marine fish. The results provide new clues for further research on the molecular mechanisms underlying hypoxia tolerance in L. crocea.

Keywords: hypoxia; transcriptome; WGCNA; hub genes; Larimichthys crocea

\section{Introduction}

Oxygen is a key environmental factor for growth, development, and reproduction of various living organisms; however, as it is not easily soluble in water, the dissolved oxygen (DO) content of natural water is exceptionally low ( 1/34th of that in air). Furthermore, the DO levels in water bodies are susceptible to various natural and human-induced 
factors, resulting in aquatic animals often suffering from hypoxia effects on their growth, development, and reproduction [1,2]. Today, hypoxia has become one of the most serious and widespread problems in freshwater and marine ecosystems worldwide. Studies show that more than 400 hypoxia zones have emerged in the world's oceans, covering thousands of square kilometers, with some offshore areas even becoming permanently hypoxic. Moreover, environmental factors such as climate change are set to exacerbate the problem [3-6]. Hypoxia has altogether caused massive mortality of invertebrates and fish in global waters, altered migration and reproductive cycles, and reduced habitat areas, which seriously affect the stability of the earth's ecosystem [7]. Imperatively, the effects of hypoxia on aquatic animals have become a hot topic in academic research worldwide.

The large yellow croaker (Larimichthys crocea), a marine fish endemic to the southeast coast of China, is named after the croak it makes when spawning. Sought after for its delectable taste and high nutritional value, artificial breeding of $L$. crocea was achieved in 1985, therefore increasing its breeding area and production to become the largest cultured marine fish in China [8]. The limited tolerance of L. crocea to hypoxia, especially in net cage culture environments, usually results in significant mortality and huge economic losses [9-11]. It is thus vital to investigate the molecular mechanisms underlying hypoxia stress in L. crocea to both elucidate its adaptative mechanisms to DO changes and inform the development of selective breeding for hypoxia resistance.

Quantitative real-time PCR (qRT-PCR) and microarray techniques have been widely used for gene expression assessment in aquatic genomics studies [12-14]. However, compared to these techniques, the high-throughput RNA sequencing approach (RNA-seq) provides more accurate quantification of gene expression and a view of the entire transcriptome $[15,16]$. RNA-seq data are now widely used in gene expression studies in aquatic animals and have also been successfully applied in gene co-expression network analysis [17-19]. Weighted gene co-expression network analysis (WGCNA) is a method frequently used to explore the complex relationships between genes and phenotypes. In WGCNA, genes with similar expression patterns are likely to be functionally related or members of the same pathway, with specific physiological significance for which hub regulatory genes can be identified [20]. This method has been successfully used to model acute infection of Pseudomonas plecoglossicida with L. crocea [21]; however, RNA-seq data and co-expression network analysis have not been applied in studies related to hypoxia in L. crocea.

In this study, transcriptome samples from different time points and tissues of $L$. crocea under hypoxic stress were used for constructing a co-expressed gene network using WGCNA analysis. In addition, each module gene with the samples was correlated with uncover the hub genes related to hypoxia regulation, with the aim of providing new clues for further research on the molecular genetic mechanisms of hypoxia tolerance in L. crocea.

\section{Results}

\subsection{Illumina Sequencing and Reads Mapping}

To explore the changes in L. crocea under hypoxia and reoxygenation conditions, the transcriptomes of blood, gills, and liver of $L$. crocea under different hypoxia and reoxygenation stress times $(0,6,24,48,96$, and $120 \mathrm{~h}$ ) were sequenced. A total of 956,902,216 clean reads were obtained from 18 transcriptome samples by sequencing with the Illumina 2500 platform (Supplementary Table S1). The number of clean bases was approximately $142.97 \mathrm{~Gb}$ and the amount of mean clean data of each sample was $7.94 \mathrm{~Gb}$. The GC content of the 18 samples was between $43.78 \%$ and $50.73 \%$, and the base GC content basically conformed to the normal distribution. More than $95.92 \%$ of the bases had a base accuracy of $99 \%$, and more than $91 \%$ of bases had a base accuracy of $99.9 \%$. The clean reads of each sample were sequenced and compared with the reference genome of $L$. crocea. The mapping rates from the 18 transcriptome libraries were high, ranging from $76.35 \%$ to $86.76 \%$. These results indicate the quality of the transcriptome sequencing data and validate its use in the subsequent data analysis. 


\subsection{Correlation Analysis between Samples}

Principal component analysis (PCA) results of transcriptome samples indicated that the tissue type is the main factor affecting transcriptome results. Sequenced samples were first divided into three sub-categories based on the three tissue types, and then divided into two categories ( $0 \mathrm{~h}-6 \mathrm{~h}-24 \mathrm{~h}$ and $48 \mathrm{~h}-96 \mathrm{~h}-120 \mathrm{~h}$ ) based on the time points (Figure 1A). The main expression of the transcriptomes of the 18 samples is shown in Figure 1B. The heat map shows clustering results similar to those of the PCA analysis.

A

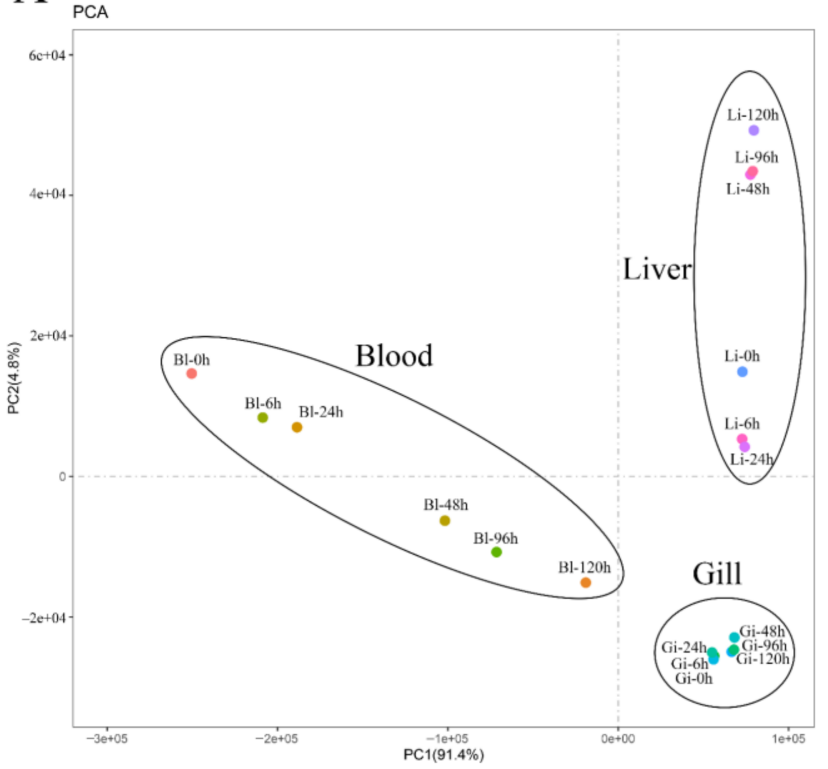

$\mathrm{B}$

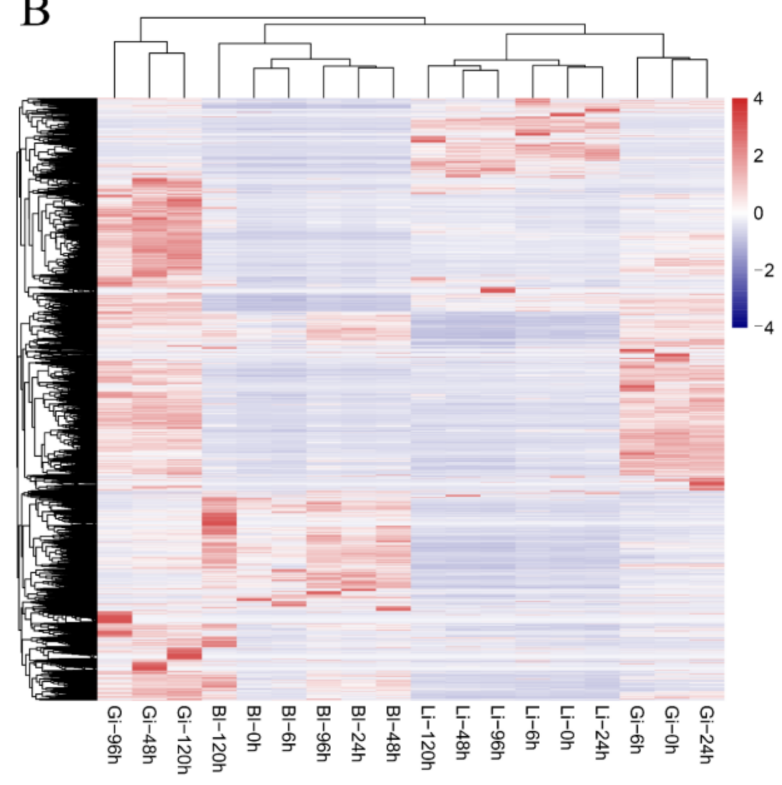

Figure 1. Relationship analysis between samples of different tissues under hypoxia and reoxygenation conditions in $L$. crocea. (A) Principal component analysis (PCA) of different transcriptome samples; (B) Heat maps analysis of all expressed genes in different samples, values in toolbar are log10 FPKM values of expressed genes.

\subsection{Differentially Expressed Genes (DEGs)}

To analyze the dynamic changes in L. crocea during hypoxia and reoxygenation, multiple transcriptome comparisons and subsequent in-depth DEG analyses were performed. In this experiment, $0 \mathrm{~h}$ samples of each tissue (liver: Li- $0 \mathrm{~h}$; gills: $\mathrm{Gi}-0 \mathrm{~h}$; and blood: Bl-0 h) were used as the control group; and compared to samples of other time points to screen for DEGs. A total of 14,881 DEGs were screened from 15 analysis groups (Figure 2). There were 4037, 6203, and 4641 DEGs in the blood, gills, and liver, respectively (Supplementary Table S2). The specific numbers of DEGs in each group are shown in Figure 2.

To carry out an in-depth analysis of DEGs, Gene Ontology (GO) and Kyoto Encyclopedia of Genes and Genomes (KEGG) enrichment analysis of the DEGs were performed. In blood, the majority of the five GO terms with the most significant enrichment are related to the function of the nucleosome, as well as the combination and transport of oxygen. The five most significant GO terms enriched in the gills were related to the regulation of osmotic pressure or ion transport, in addition to the combination and transport of oxygen. However, in the liver, after analyzing the first five GO terms that were significantly enriched at various times, it was found that most of the DEGs were enriched in processes related to energy metabolism, as well as immune and antioxidant systems (Supplementary Figure S1). 


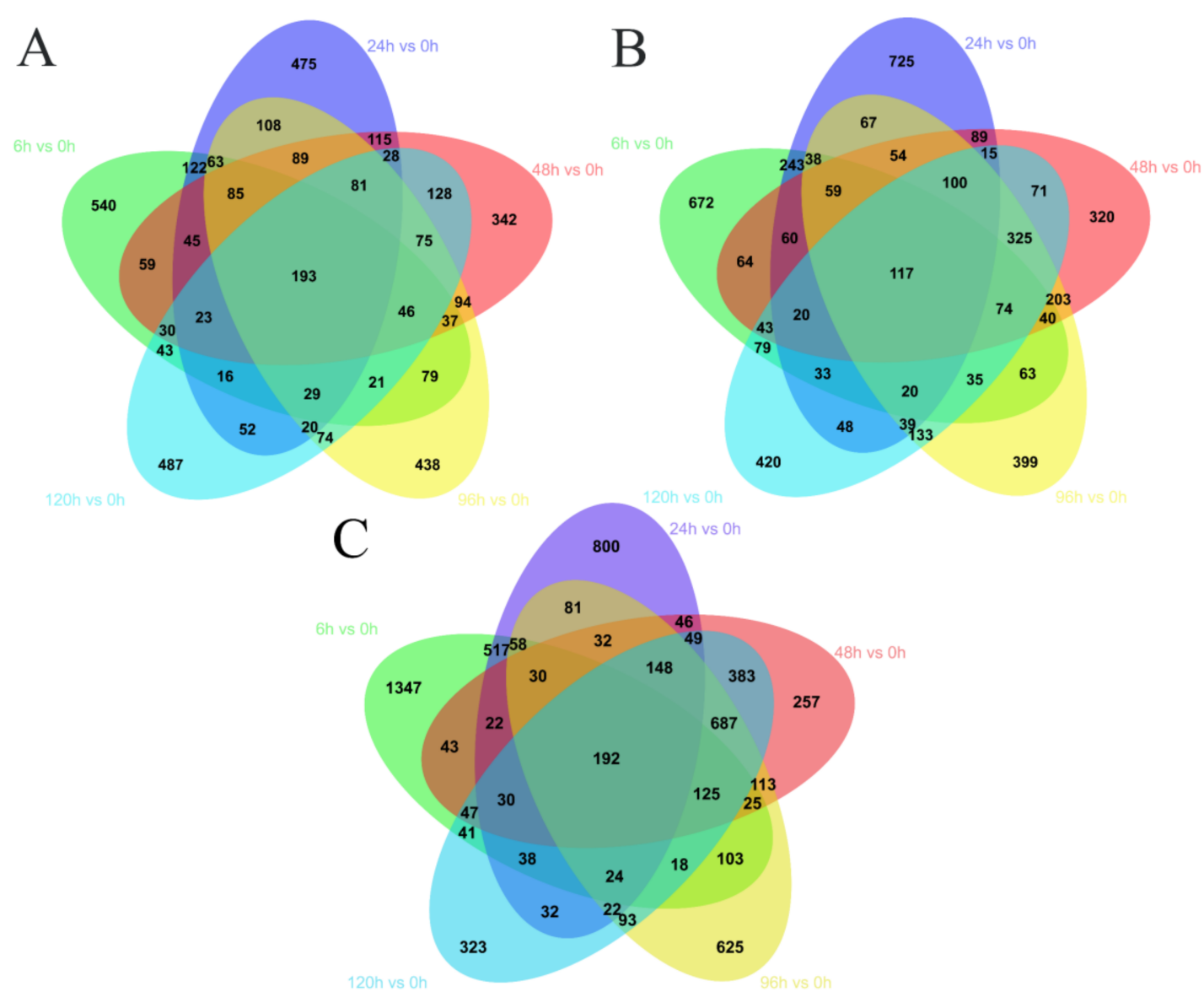

Figure 2. Venn diagrams of differentially expressed genes in the blood (A), liver (B), and gills (C) of L. crocea under hypoxia and reoxygenation conditions. All samples were compared to the $0 \mathrm{~h}$ control for the respective tissue types.

The KEGG enrichment results showed that a total of nine significantly up or downregulated pathways were enriched in blood; among which the ribosome pathway was significantly up-regulated at each time point; lipid metabolism-related pathways were significantly down-regulated at Bl-6 $\mathrm{h}$ and $\mathrm{Bl}-24 \mathrm{~h}$; and protein metabolism-related pathways were also significantly down-regulated at Bl-48 h and Bl-96 h (Supplementary Figure S2). For the DEGs in the gills of L. crocea, 23 pathways with significant changes were enriched, including eight pathways related to signal transduction, nine pathways related to metabolism, and some related to immune factors and cell cycle (Supplementary Figure S3). Among them, the ECM-receptor interaction was significantly up-regulated at all time points except Gi-24 h. Most of the pathways that were enriched in the liver were related to carbohydrate metabolism, lipid metabolism, and protein metabolism (Supplementary Figure S4).

\subsection{WGCNA}

WGCNA was performed to identify genes related to hypoxic stress. After filtering out the low-expressed genes (fragments per kilobase of transcript per million; FPKM $<0.01$ ) in 18 transcriptome sequencing libraries, 18,054 effective genes were obtained for WGCNA analysis. The WGCNA packet was used to calculate the weight value to make the network conform to the scale-free network distribution. According to the results in Figure 3A, the soft threshold power $\beta=8$ was selected to construct a co-expression network. The gene co-expression network was then successfully constructed and divided into 21 co-expression modules, with each module using a different color to represent the genes clustered together (Figure 3B). The number of genes varied considerably between modules, with the black module clustering to the highest number of genes (8572) while the orangered4 module contained the fewest (109) (Figure 3D) (Supplementary Table S3). 
A
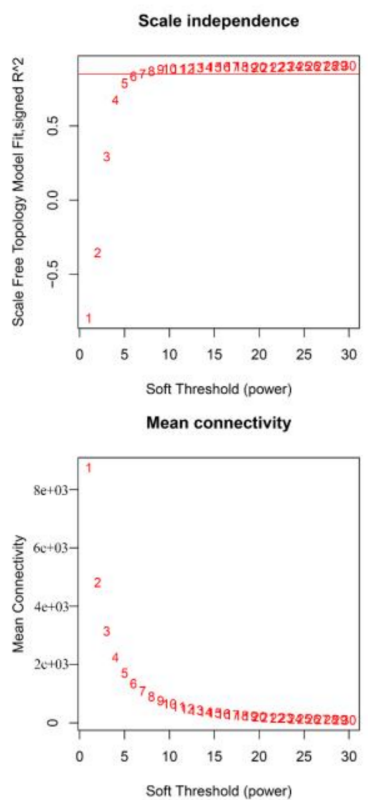

C

MEorangered4
MEdarkmagenta
MEdarkorange
MEviolet
MEpaleturquoise
MEbrown
MElightcyan
MEmagenta
MEplum1
MEskyblue3
MEsaddlebrown
MEdarkgreen
MEdarkturquoise
MEdarkred
MEblack
MEsalmon
MEsteelblue
MEdarkgrey
MEwhite
MEdarkolivegreen
MEtan

Module-trait relationships

$0080.017-0.06-0.06-0.06-0.07-0.05-0.07-0.093-0.12-0.11-0.09-0.081-0.11$ $0.0260 .0670 .190 .10 .86022-0.08-0.07-0.08-0.08-0.04-0.087-0.16-0.17-0.18-0.19-0.13-0.18$ $(0.9)(0.8)(0.4)(0.7)(4-06)(0.4)(0.7)(0.8)(0.7)(0.7)(0.9)(0.7)(0.5)(0.5)(0.5)(0.5)(0.6)(0.4)$ 0.0470011-0.03-0.0070017 0.99-0.064-0.04-0.06-0.03-0.04-0.053-0.1-0.11-0.1-0.12-0.083.0.1. $0.0470 .011-0.03-0.007 .017$
$(0.9)$ (1) $(0.9)$ (1) $(0.9)(6-99-14 \times 0.8)(0.9)(0.8)(0.9)(0.9)(0.8)(0.7)(0.7)(0.7)(0.6)(0.7)(0.7)$ $0.02,00530.96 \quad 0.12-0.0220 .079-0.06-0.04-0.04-0.05-0.03-0.069-0.12-0.14-0.12-0.15-0.13-0.14$

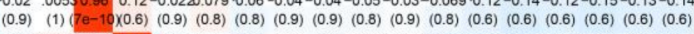
$0.0430 .077-0.0490 .98-0.05$
$(0.9)(0.8)(0.8)(6-13)(0.8)(1)(0.8)(0.9)(0.8)(0.9)(0.9)(0.7)(0.7)(0.6)(0.7)(0.7)(0.7)(0.7)$ $\begin{array}{lllllllll}0.13 & 0.41 & 0.33 & 0.39 & 0.11 & 0.4 & -0.070 .008-0.02-0.01-0.01-0.058-0.25-0.28-0.26-0.28-0.24-0.28\end{array}$ $(0.6)(0.09)(0.2)(0.1)(0.7)(0.1)(0.8)$ (1) (0.9) (1) (1) $(0.8)(0.3)(0.3)(0.3)(0.3)(0.3)(0.2)$ $0.0990 .89-0.0520 .065-0.110 .02-0.00660 .120 .0360 .092 \quad 0.10 .027-0.14-0.18-0.17-0.19-0.21-0.2$ $(0.7 \times 8 \mathrm{~B}-07 \times 0.8)(0.8)(0.7)(0.9)(1)(0.6)(0.9)(0.7)(0.7)(0.9)(0.6)(0.5)(0.5)(0.4)(0.4)(0.4)$ $0.091-0.05-0.07-0.06-0.09-0.066-0.02-0.0150 .04-0.0210 .013-0.99-0.07-0.083-0.08-0.08-0.06-0.07$ $(0.7)(0.8)(0.8)(0.8)(0.7)(0.8)(0.9)$ (1) $(0.9)(0.9)$ (1) (30-16 $\times 0.8)(0.7)(0.8)(0.8)(0.8)(0.8)$ $-0.17-0.11-0.14-0.14-0.15-0.13-0.06-0.04-0.06-0.02-0.020 .00610 .020 .96-0.020 .03-0.00110 .1$ (0.5) (0.7) (0.6) (0.6) (0.6) (0.6) (0.8) (0.9) (0.8) (0.9) (0.9) (1) $(0.9)(\mathrm{e}-10 \times(0.9)(0.9)$ (1) $(0.7)$

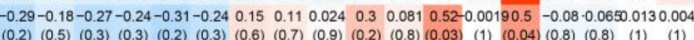
(0.2) $(0.5)(0.3)(0.3)(0.2)(0.3)(0.6)(0.7)(0.9)(0.2)(0.8)(0.03)(1)(0.04)(0.8)(0.8)$ (1) (1)

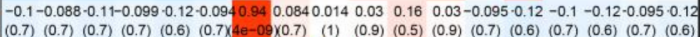
(0.7) $-0.11-0.077-0.1-0.075-0.1-0.08-0.0150 .029$
$0.9880 .0410 .0580 .004-0.07-0.09-0.08-0.09-0.08-0.09$
$(0.7)(0.8)(0.7)(0.8)(0.7)(0.7)(1)(0.9)(6 e-12 \times 0.9)(0.8)(1)(0.8)(0.7)(0.7)(0.7)(0.7)(0.7)$

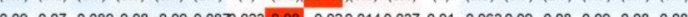

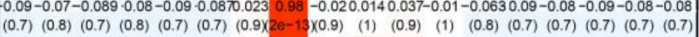
(1) $-0.0950 .07-0.09-0.079-0.1-0.08-0.0140 .0560 .00130 .970 .095-0.051-0.07-0.1-0.08-0.08-0.09-0.09$
$(0.7)(0.8)(0.7)(0.8)(0.7)(0.7)(1)(0.8)(1)(80-12)(0.7)(0.8)(0.8)(0.7)(0.7)(0.7)(0.7)(0.7)$

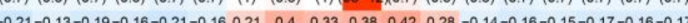

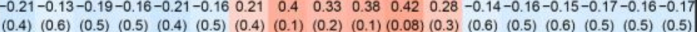
(0.4) (0.6) (0.5) (0.5) (0.4) (0.5) (0.4) $(0.1)(0.2)(0.1)(0.08)(0.3)(0.6)(0.5)(0.6)(0.5)(0.5)(0.5)$

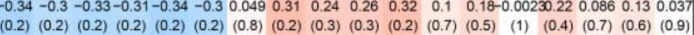
(0.2) $0.09-0.07-0.09-0.082-0.1-0.07-0.06-0.04-0.05-0.06-0.04-0.0610 .026-0.05-0.04-0.03-0.0360$
$(0.7)(0.8)(0.7)(0.7)(0.7)(0.8)(0.8)(0.9)(0.8)(0.8)(0.9)(0.8)(0.9)(0.8)(0.8)(0.9)(0.9)(2 e-1$

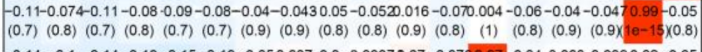

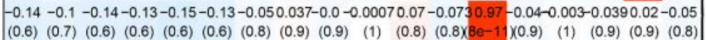
$-0.15-0.12-0.15-0.12-0.15-0.13-0.07-0.008-0.03-0.03-0.01-0.0860 .088-0.0390 .960 .0230 .0250 .02$

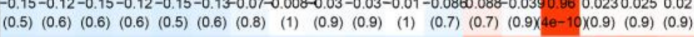
$-0.2-0.18-0.2-0.19-0.21-0.2-0.16-0.13-0.14-0.14-0.12-0.150 .350 .23 \quad 0.4 \quad 0.350 .38 \quad 0.31$
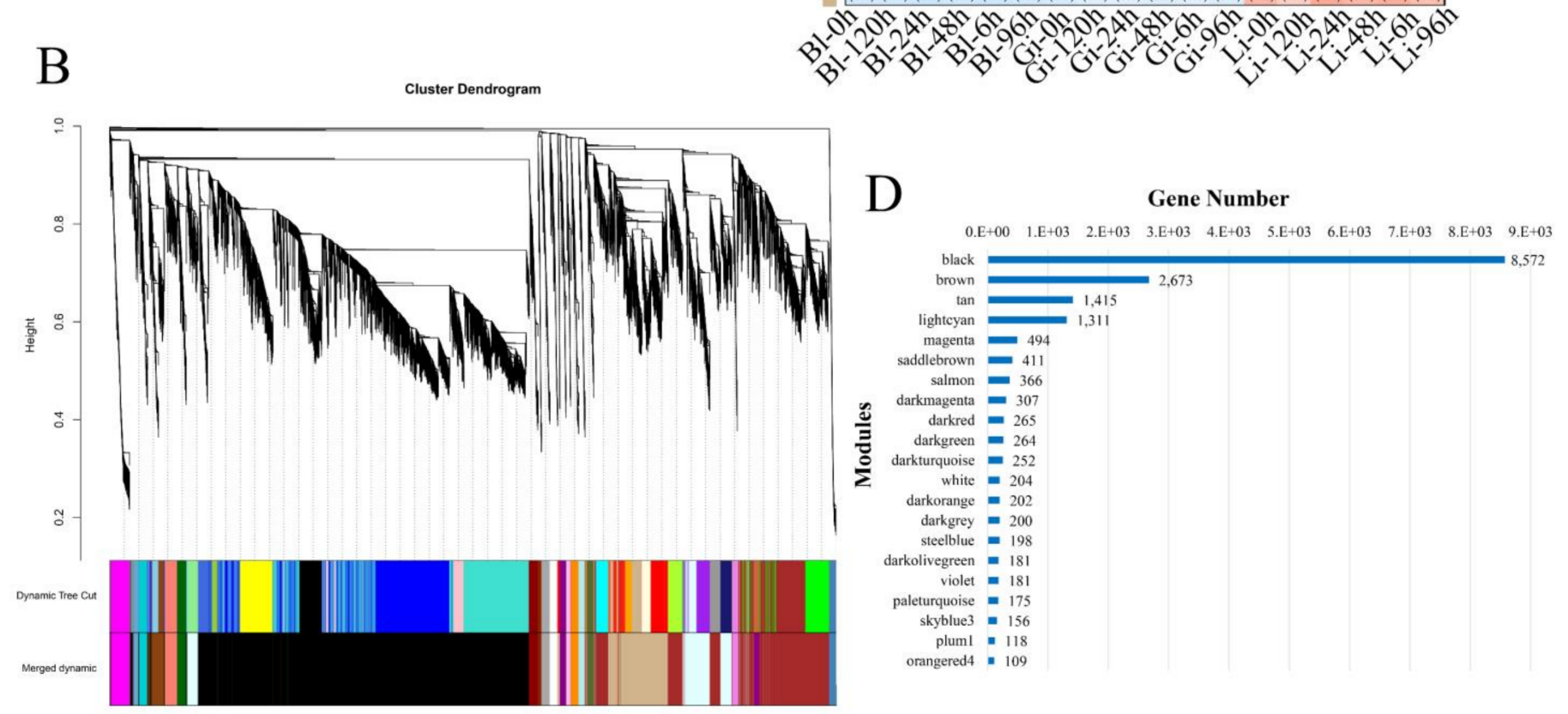

Figure 3. Weighted gene co-expression network analysis (WGCNA) of gene expressions. (A) Scale-free topology model fit and gene mean connectivity under different soft threshold power; (B) Clustering dendrogram of genes and module division by WGCNA. The 21 co-expression modules are shown in different colors; (C) Heat map for the association of gene co-expression network modules with samples; (D) Histogram of the number of genes in each module.

To identify modules that were significantly associated with hypoxia, module eigengenes was calculated using all genes in each module and then correlated the signature genes with the 18 samples for association analysis (Figure 3C). The closer the correlation between a sample and a module is to the absolute value of 1 , the more likely it is that the trait is related to the genetic function of the module. The correlation analysis showed that the orangered4, darkorange, violet and paleturquoise modules were significantly and positively correlated with Bl-0 h, Bl-96 h, Bl-24 h and Bl-48 h, respectively $\left(\mathrm{R}^{2}>0.9\right.$, 
$p<0.01)$. The saddlebrown, darkgreen, darkturquoise, darkred, and magenta modules were significantly and positively correlated with Gi-0 h, Gi-24 h, Gi-120 h, Gi-48 h and Gi$96 \mathrm{~h}$, respectively $\left(\mathrm{R}^{2}>0.9, p<0.01\right)$. The plum1, steelblue, darkgrey, white, and olivegreen modules were significantly and positively correlated with Li-120 h, Li-96 h, Li-6 h, Li-0 h, and Li-24 h, respectively $\left(R^{2}>0.9, p<0.01\right)$.

\subsection{Hub Genes Selections}

To elucidate the biological functions of the different modules in hypoxic stress, GO functional enrichment analysis and KEGG pathway enrichment analysis were performed for the 14 modules that were significantly associated with the samples in the previous section.

The top 10 enriched terms for each module are shown in Figure 4. The genes in the darkorange module were mainly enriched in four categories: intercellular communication (regulation of cell migration, cell adhesion, tight junction), oxidation-reduction reactions (oxidoreductase activity, cell redox homeostasis), gene replication (nucleosome, RNA-dependent DNA replication, RNA-directed DNA polymerase activity), and other activities (olfactory receptor activity, regulation of endopeptidase activity). Most of the genes in the magenta module are related to ion regulation (potassium ion transmembrane transporter activity, intracellular ligand-gated ion channel activity, calcium ion transport into cytosol, cytosolic calcium ion transport, release of sequestered calcium ion into cytosol, sequestering of calcium ion, negative regulation of sequestering of calcium ion, potassium ion transmembrane transport, and cellular potassium ion transport). Most of the genes in the saddlebrown and darkolivegreen modules are associated with energy metabolism. Some of the other related modules were not significantly enriched $(p>0.05)$ for the KEGG pathway or did not identify effective hub genes, and were therefore excluded for subsequent in-depth analysis.
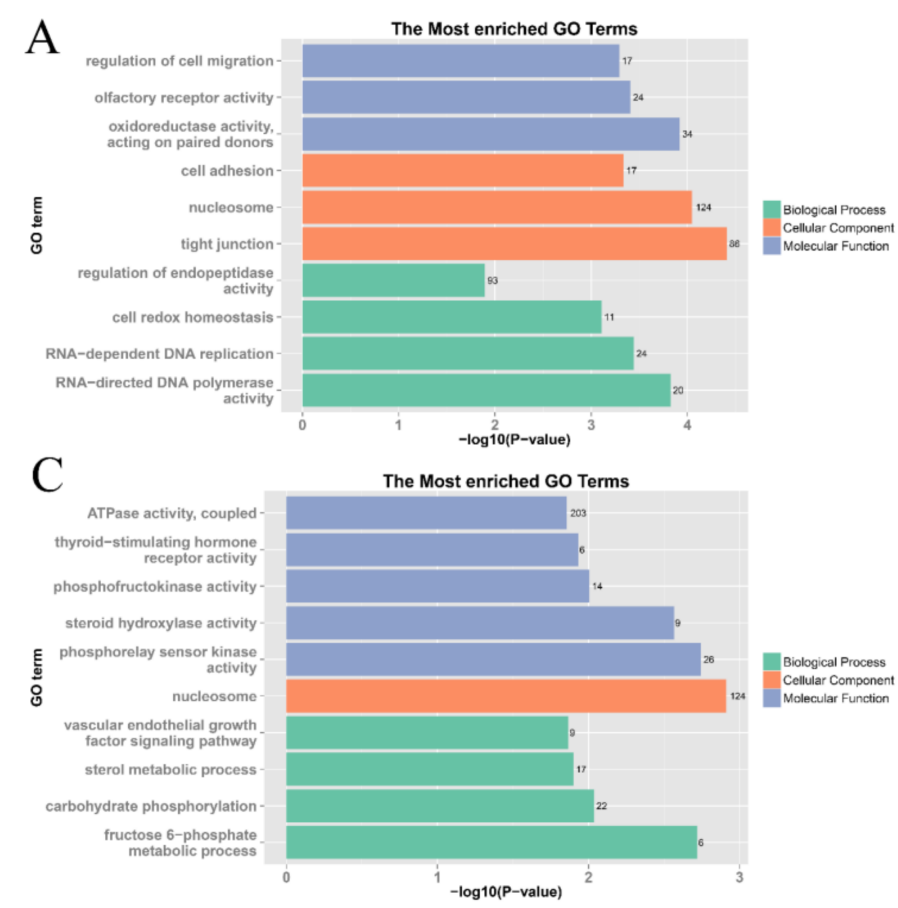

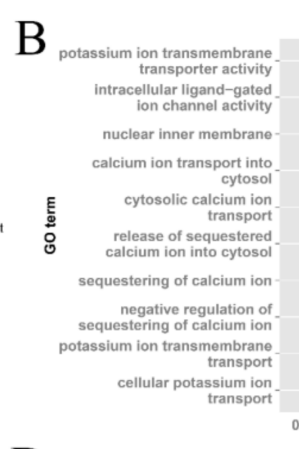

$\mathrm{D}$

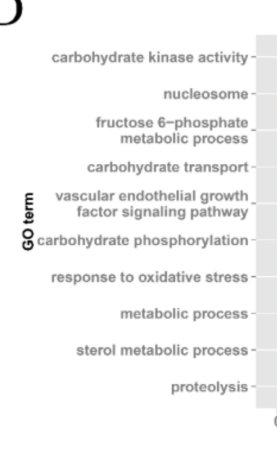

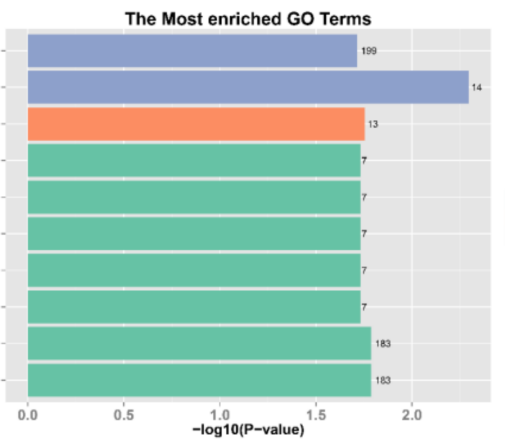

The Most enriched Go Terms

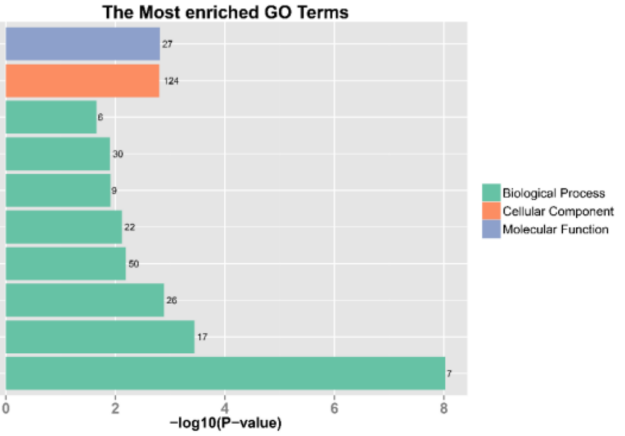

Figure 4. Gene ontology (GO) enrichment analysis of genes within highly correlated modules. (A) darkorange module; (B) magenta module; (C) saddlebrown module; (D) darkolivegreen module.

The annotation results of the four modules compared to the KEGG database are presented in Figure 5. The enrichment results of pathways showed that the focal adhesion, apoptosis, mammalian target of rapamycin (mTOR) signaling pathway, insulin signaling pathway, ribosome, and VEGF signaling pathways were significantly enriched in the dark- 
orange module. In the magenta module, functions relating to drug metabolism-cytochrome P450, nitrogen metabolism, ribosome, p53 signaling pathway, PPAR signaling pathway, and cell cycle were significantly enriched. In the saddlebrown module, arginine and proline metabolism, fructose and mannose metabolism, purine metabolism, biosynthesis of amino acids, pentose phosphate pathway, adrenergic signaling in cardiomyocytes, carbon metabolism, glycolysis/gluconeogenesis, and calcium signaling pathways were significantly enriched. In the darkolivegreen module, most of the enrichment occurs in metabolism and signaling-related pathways.
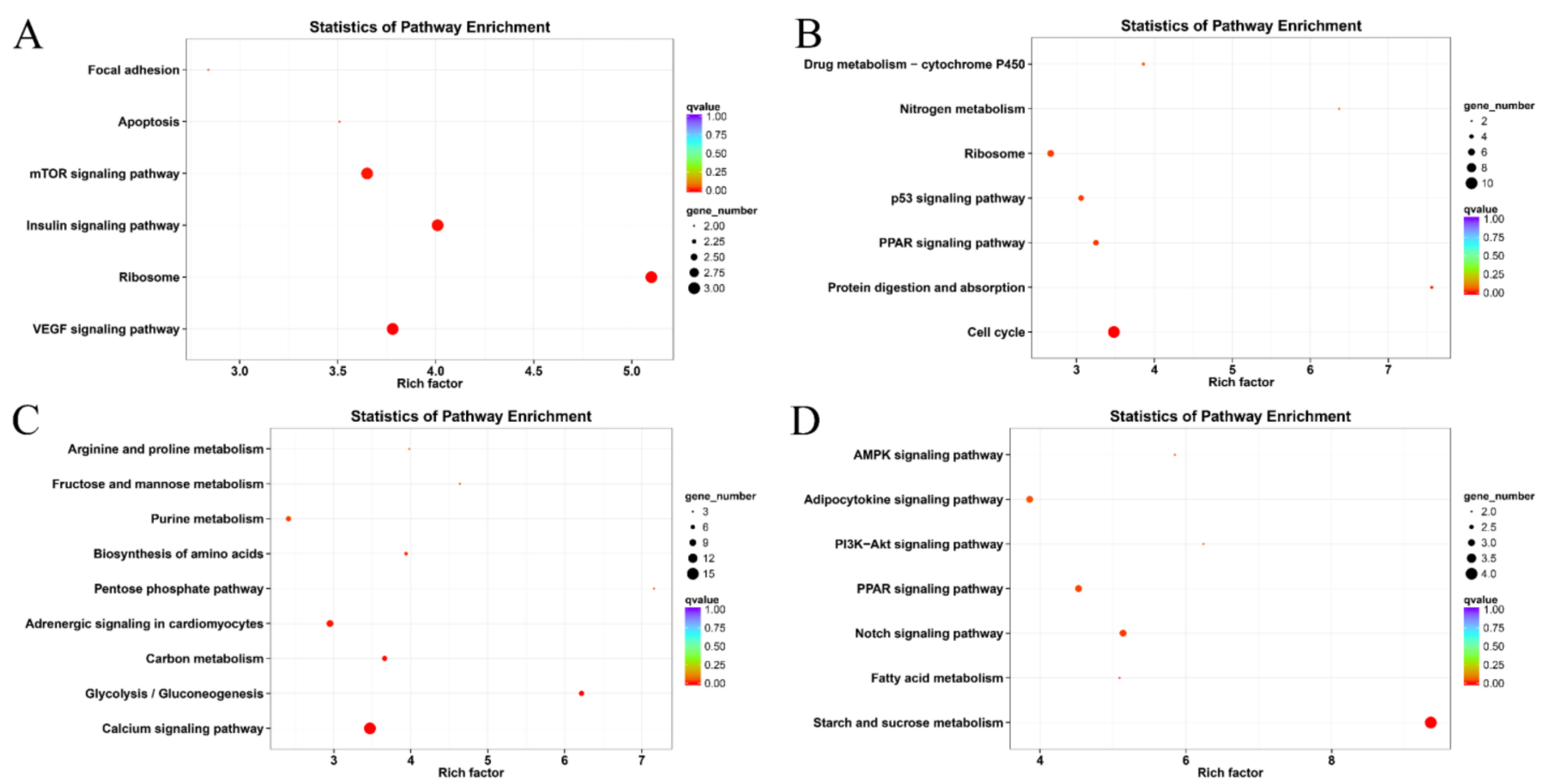

Figure 5. Kyoto Encyclopedia of Genes and Genomes enrichment analysis of genes within highly correlated modules. (A) darkorange module; (B) magenta module; (C) saddlebrown module; (D) darkolivegreen module.

To further determine the function of co-expressed genes within each module and to study hub genes, gene co-expression networks was constructed using Cytoscape (version 3.7.2) software for four selection modules, including darkorange, magenta, saddlebrown, and darkolivegreen modules. The highest degree of co-expressed genes (hub genes) was illustrated with a bigger size and darker color (Figure 6) (Table 1). For example, in the darkorange module, 60S ribosomal protein L8 (RPL8), 40S ribosomal protein S16 (RPS16), iron-sulfur cluster assembly 1 (ISCA1), and erythroid differentiation-related factor 1 (EDRF1) were identified as hub genes. In the magenta module, potassium channel subfamily K member 5 (KCNK5), sodium-coupled neutral amino acid transporter 2 (SNAT2), solute carrier family 22 member 7 (SLC22A7) and kinesin-like protein KIF3B (KIF3B) were recognized as hub genes. In the saddlebrown module, phosphofructokinase, liver type

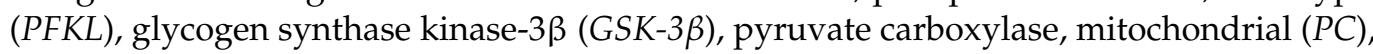
and alcohol dehydrogenase $[\mathrm{NADP}(+)] \mathrm{A}$ isoform $\mathrm{X} 1(A K R 1 A 1)$ were recognized as hub genes. In the darkolivegreen module, acetyl-CoA carboxylase alpha $(A C A C A)$, heat shock protein $90 \mathrm{kDa}$ beta member 1 (HSP90B1), and phosphatidylinositol 4,5-bisphosphate 3-kinase catalytic subunit delta (PIK3CD) were identified as hub genes. 
A

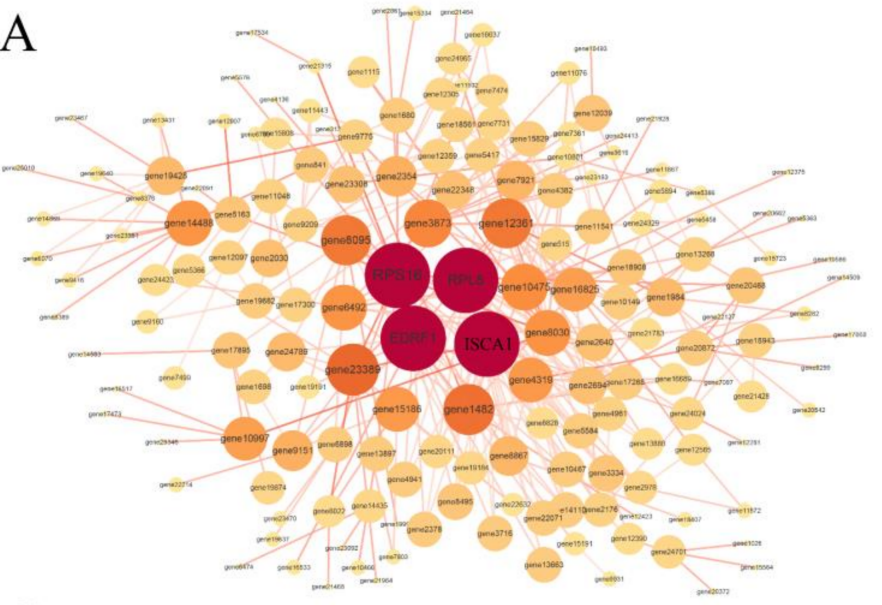

$\mathrm{C}$

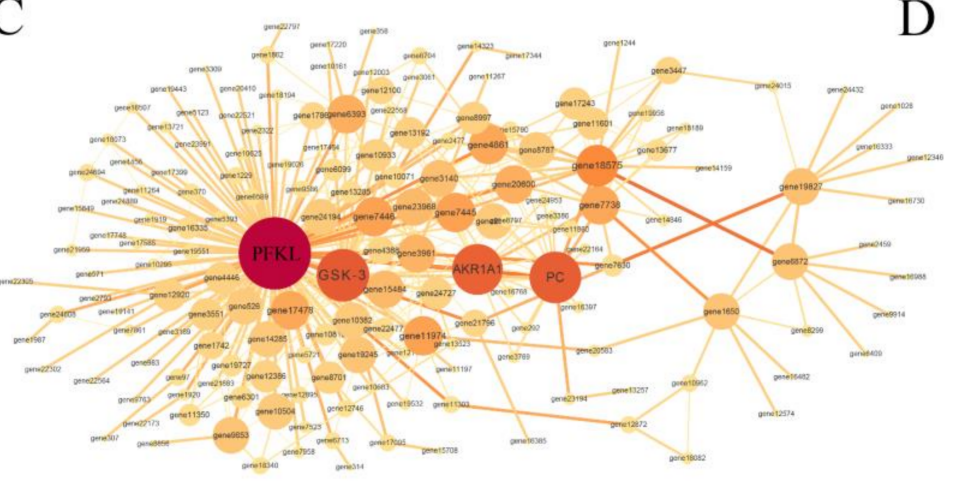

$\mathrm{B}$

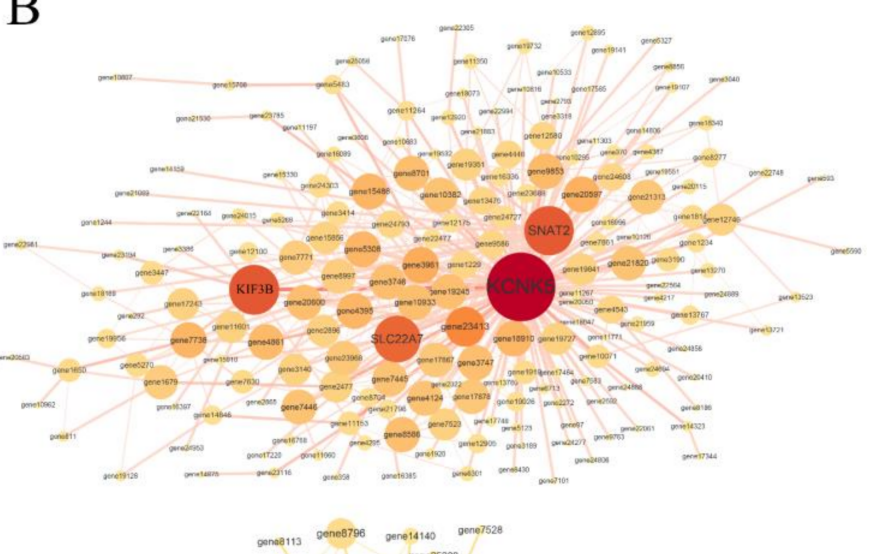

D

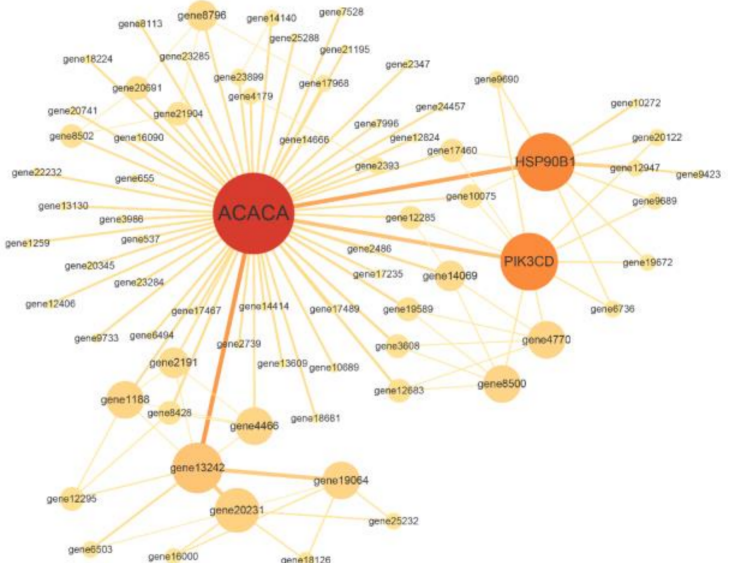

Figure 6. The local co-expression network analysis of darkorange (A) magenta (B) saddlebrown (C), and darkolivegreen (D) modules. The size and color of each node circle is positively correlated with the number of interacting genes.

Table 1. Functional annotation of the hub genes of the target module.

\begin{tabular}{|c|c|c|c|c|}
\hline Module & Gene Symbol & Gene ID & Description & Degree \\
\hline darkorange & RPS16 & EH28_19390 & $40 S$ ribosomal protein $\mathrm{S} 16$ & 42 \\
\hline darkorange & ISCA1 & EH28_11232 & Iron-sulfur cluster assembly 1 & 38 \\
\hline darkorange & EDRF1 & EH28_15699 & Erythroid differentiation-related factor 1 & 37 \\
\hline darkorange & RPL8 & EH28_17632 & $60 S$ ribosomal protein $\mathrm{L} 8$ & 37 \\
\hline magenta & KCNK5 & EH28_04734 & Potassium channel subfamily K member 5 & 118 \\
\hline magenta & SNAT2 & EH28_19256 & Sodium-coupled neutral amino acid transporter 2 & 58 \\
\hline magenta & KIF3B & EH28_23591 & Kinesin-like protein KIF3B & 56 \\
\hline magenta & SLC22A7 & EH28_16974 & Solute carrier family 22 member 7 & 48 \\
\hline saddlebrown & PFKL & EH28_06053 & phosphofructokinase, liver type & 97 \\
\hline saddlebrown & GSK-3 $\beta$ & EH28_10886 & Glycogen synthase kinase- 3 beta & 42 \\
\hline saddlebrown & $P C$ & EH28_15368 & Pyruvate carboxylase, mitochondrial & 40 \\
\hline saddlebrown & AKR1A1 & EH28_24096 & Alcohol dehydrogenase [NADP $(+)]$ A isoform X1 & 38 \\
\hline darkolivegreen & $A C A C A$ & EH28_08273 & Acetyl-CoA carboxylase alpha & 56 \\
\hline darkolivegreen & HSP90B1 & EH28_10845 & Heat shock protein $90 \mathrm{kDa}$ beta member 1 & 20 \\
\hline darkolivegreen & PIK3CD & EH28_12563 & $\begin{array}{l}\text { Phosphatidylinositol 4,5-bisphosphate 3-kinase } \\
\text { catalytic subunit delta }\end{array}$ & 19 \\
\hline
\end{tabular}

\subsection{Quantitative PCR Validation}

To verify the reliability of the transcriptomic data, eight DEGs were selected for qRT-PCR validation, namely citrate synthase (CS), glycogen synthase kinase-3 $\alpha(G S K 3 \alpha)$, hexokinase-1 (HK1), phosphoenolpyruvate carboxykinase (PEPCK), 6-phosphofructokinase liver type (PFKL), succinate dehydrogenase complex subunits B $(S D H B)$, lactate dehydrogenase A ( LDHA), and triosephosphate isomerase (TPI). As shown in Supplementary 
Figure S5, the qRT-PCR results were generally consistent with the gene expression patterns of the transcriptomic data. Furthermore, correlation analysis also showed a highly significant linear correlation between the results of qRT-PCR and RNA-seq $\left(R^{2}=0.7952\right)$, indicating the high reliability of the transcriptomic data (Figure 7).

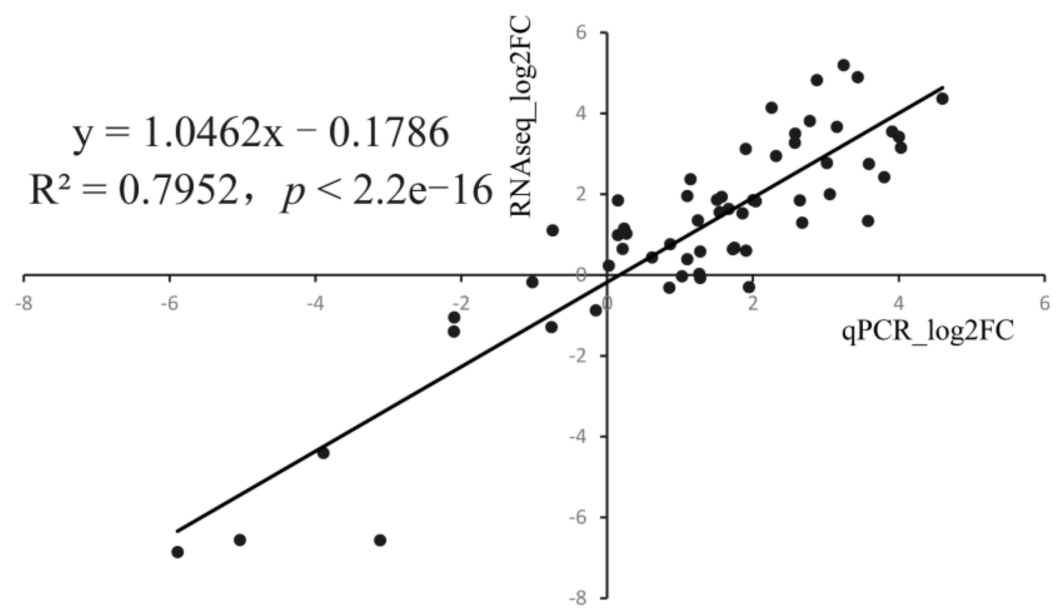

Figure 7. Correlation analysis of Quantitative real-time PCR (qRT-PCR) and RNA sequencing (RNAseq) results.

\section{Discussion}

The changes in gene expression in L. crocea when exposed to acute hypoxia stress are complex, and transcriptome sequencing of these events has not been fully investigated [8,9]. In this study, transcriptome sequencing was used to analyze gene expression in L. crocea at different time points and in different tissues (liver, blood, and gills) under hypoxia and reoxygenation stress, and constructed gene co-expression networks together with performing gene network interaction analysis on four of the modules to find the hub genes. The results indicate that hub genes associated with hypoxia and reoxygenation stress may play important roles in identifying candidate biomarkers and understanding the molecular mechanisms of hypoxia tolerance.

\subsection{Effects of Hypoxia and Reoxygenation on the L. crocea Transcriptome}

The PCA and heat map analysis of the three tissues at each time point of hypoxia and reoxygenation stress showed that all three tissues exhibited significant clustering by tissue type rather than time points with significant variation among the samples of each tissue. The results of GO and KEGG enrichment analysis showed that the adaptation strategy of L. crocea to hypoxia was significantly tissue-specific, with blood, gills, and liver associated with functional terms and pathways of oxygen transport, ion regulation, and energy metabolism, respectively, which corresponded to the physiological functions of the tissues as well. Similarly, transcriptome analysis of liver, gills, and kidney tissues of Leuciscus waleckii under alkaline stress showed different patterns of energy metabolism in different tissues, and DEGs were specific among tissues [22]. The adaptation of gills, liver and muscle tissues of Cynoglossus semilaevis to a high-temperature environment was also significantly different, and there was obvious tissue specificity for certain biological functions [23]. The tissue specificity exhibited under hypoxia and reoxygenation stress is mainly due to the presence of tissue-specific genes (luxury genes). However, further investigation is required to explore this in more depth.

Based on the tissue-specific analysis of hypoxia adaptation in L. crocea, we expanded on the different functions of the three tissues in hypoxia adaptation. GO analysis and KEGG enrichment analysis were performed on a total of 14,881 DEGs from three tissues, and among these GO terms and pathways, metabolism-related biological process terms were the most enriched, including "carbohydrate metabolism", "lipid metabolism" and 
"amino acid metabolism", followed by oxygen transport-related biological terms in gills and blood, including "oxygen transport", "hemoglobin complex", and "transferrin transport". At the same time, important processes such as "signaling" and "osmolarity regulation" occur frequently. Therefore, this section discusses four aspects of energy metabolism, signal transduction, oxygen transport, and osmotic regulation to provide a comprehensive account of the molecular regulatory mechanisms in L. crocea under hypoxia and reoxygenation conditions. As energy metabolism-related topics have been covered in detail in previous articles [9], this discussion will only brush over the most important aspects.

Signal transduction enhances the body's tolerance to hypoxia by activating transcription or inhibiting translation, which is of great significance for the adaptation of aquatic animals to hypoxia $[2,24,25]$. This study found that signaling pathways such as the transforming growth factor- $\beta$ (TGF- $\beta$ ) signaling pathway, insulin signaling pathway, glucagon signaling pathway, ECM-receptor interaction, focal adhesion, and tight junctions were significantly enriched. TGF- $\beta$ is a signaling pathway mediated by transforming growth factors, which maintains the stability of the cell environment and promotes embryonic development, tissue repair, cell proliferation and differentiation, and immune regulation [26]. It also plays a key role in regulating cell proliferation and is of great significance for maintaining the environmental homeostasis of $L$. crocea tissue under hypoxia [27]. The insulin signaling pathway plays an important role in carbohydrate and lipid metabolism. It can accelerate the transport of glucose into cells, promote the storage of intracellular triglycerides and glucose in the form of glycogen in various tissues, inhibit glycogen decomposition and gluconeogenesis, and maintain the balance of carbohydrate and lipid metabolism [28,29]. Furthermore, glucagon and insulin induce opposite metabolic effects [30]. Therefore, the tissues of $L$. crocea under hypoxia can jointly regulate the expression of genes and proteins related to glycolipid metabolism through the insulin signaling pathway and glucagon signaling pathway, and alter the level of glycolipid metabolism in tissue cells to adapt to the altered metabolic pattern caused by hypoxic stress. Moreover, the ECM is a complex mixture of structural and functional macromolecules that plays an important role in the morphogenesis of tissues and organs as well as maintaining the structure and function of cellular tissues [31,32]. It can interact with a variety of cell surface receptors simultaneously and plays a very important role in intercellular signal transduction. The activation of ECM receptors in a hypoxic environment triggers a series of signals in the cell to cause different gene expression, which may be a key factor for the normal function of $L$. crocea tissue under hypoxic stress [33]. Functionally similar to ECM receptors, focal adhesions and tight junctions are also a type of cell-to-cell connection and involved in the regulation of intercellular signal transduction [34,35]. The above results indicate that under acute hypoxic stress, $L$. crocea can rapidly adapt to the hypoxic environment by modulating these signal transduction pathways and altering the expression of genes related to antioxidants, cell proliferation and differentiation, signal transduction, and glycolipid metabolism in the organism by regulating their target genes.

It is generally hypothesized that the effect of hypoxia on fish ion regulation is minimal. However, some studies have shown that the ion regulation and osmotic pressure balance of fish in a hypoxic environment will be affected to a certain extent. For example, Victoria et al. found that the ion balance of Gymnocypris przewalskii was significantly disrupted after $24 \mathrm{~h}$ of acute hypoxia stress [36]; similarly, the $\mathrm{Na}^{+}$and $\mathrm{Cl}^{-}$concentrations of Eptatretus stoutii decreased significantly under hypoxic conditions [37]. The GO enrichment results in $L$. crocea gills revealed that the five most significantly enriched functional groups at each time point were related to osmotic regulation or ion transport, including ammonium transmembrane transport, zinc ion binding, and organic cation transport, suggesting that hypoxia can affect ion regulation in L. crocea gills. A central process in ion regulation is active $\mathrm{Na}^{+}$transport, and ion transport through the sodium-potassium pump on the gill epithelium is therefore energy-intensive as is the energy consumed to excrete chloride ions $[38,39]$. In this study, it was found that glycolipid metabolism-related pathways such as glycerolipid metabolism, glycolysis/gluconeogenesis, and carbon metabolism were 
significantly enriched in gills, indicating that the gills of L. crocea are greatly affected by osmotic balance under hypoxic stress, and the gills glycolipid metabolism process can provide energy support for ion transport and osmotic regulation. Regarding the effect of hypoxia on the regulation of osmotic pressure in the gills of L. crocea, further experimental research is needed on its blood ion concentration as well as specific transport enzymes (such as $\mathrm{Na}^{+} / \mathrm{K}^{+}$-ATPase).

The GO analysis of DEGs in blood revealed that $\mathrm{GO}$ terms related to oxygen transport and binding, such as the vascular endothelial growth factor (VEGF) signaling pathway, hemoglobin complex, and transferrin transport, were significantly enriched. In the gills, GO terms such as hemoglobin complexes, oxygen transport viability, and oxygen binding were also significantly enriched. VEGF is a growth factor that can specifically act on vascular endothelial cells and acts as a key signal transducer in physiological and pathological angiogenesis [40]. The binding of VEGF to its specific receptor leads to a series of different signaling pathways, resulting in the up-regulation of genes mediating the proliferation of endothelial cells and vascular permeability [41]. Studies have shown that a significant increase in the expression of VEGF under hypoxic conditions can increase oxygen-carrying capacity [42]. Furthermore, an increase in hemoglobin, a key protein for oxygen binding, in response to hypoxic conditions can also significantly increase the oxygen-carrying capacity of blood [43,44]. Additionally, transferrin (TF) is a non-hemoglobin binding iron $\beta$-globin, whose most basic physiological function is to bind and transport $\mathrm{Fe}^{3+}$. It binds to the TF receptor to maintain iron homeostasis, and absorb and transport free $\mathrm{Fe}^{3+}$ to red blood cells to synthesize hemoglobin, with the remaining $\mathrm{Fe}^{3+}$ transported to hepatocytes or macrophages for storage $[45,46]$. Studies have shown that hypoxia can promote the absorption of iron, i.e., through the up-regulation of serum TF receptor in rats exposed to hypoxia [47]. In addition, Wenger et al. found a significant increase in TF gene expression after incubating HepG2 cells under hypoxic conditions, all of which suggest that up-regulated expression of TF under hypoxic stimulation may play a critical role in hypoxia regulation [48]. Iron is an essential element in many important metabolic processes, and iron-containing proteins are required in processes such as oxygen transport, electron transfer, energy metabolism, and removal of active oxygen. Cells maintain iron homeostasis through iron absorption, storage, and use, and thus maintaining the iron homeostasis in the cell under a hypoxic environment is particularly important for the hypoxia adaptation of $L$. crocea $[47,49,50]$. As an additional mechanism influencing oxygen metabolism, erythropoietin (EPO), a colony-stimulating factor promotes the differentiation of erythroid-directed stem cells into nucleated erythroid cells capable of synthesizing hemoglobin, and secondarily stimulates the release of reticulocytes and erythrocytes from the bone marrow in vivo [51]. Under hypoxic stimulation, EPO significantly up-regulates gene expression, stimulates the proliferation and differentiation of erythrocytes, and therefore increases the oxygen-carrying capacity of the body to meet the oxygen demand in a hypoxic environment [52].

\subsection{Functional Analysis of Hub Genes}

WGCNA can cluster numerous genes into different co-expression modules according to similarities in gene expression patterns. This allows for selecting expression modules that are highly biologically relevant to the target trait, as well as constructing gene networks to mine the regulated hub genes $[53,54]$. This has proven to be an efficient data mining method in bioinformatics, and it has been used in Hexagrammos otaki, Ictalurus punctatus, Scophthalmus maximus, Oplegnathus fasciatus, Crassostrea gigas and other aquatic animals $[17-19,24,55]$. In this study, WGCNA analysis of 18,054 validated genes from 18 transcriptome samples yielded a total of 21 co-expression modules, which were correlated with the samples to select 14 modules that were significantly associated with samples for further study. GO enrichment and KEGG pathway enrichment analyses were then performed on these modules, and four modules, namely darkorange, magenta, saddlebrown and darkolivegreen, were finally obtained. 
The darkorange module contained 202 genes, of which 4 hub genes were annotated and 3 of them were DEGs in blood tissues. The hub gene of the darkorange module, ISCA1, composed of iron and sulfur ions, not only participate in energy transfer (as the auxiliary group) of electron transfer proteins, but also participate in the biochemical reactions (as the active group) of certain enzymes, redox reactions, DNA replication repair, protein translation, and other regulatory processes [56-58]. Thus, ISCA1 is a key gene in many biochemical processes and of great significance to the hypoxic adaptation of L. crocea. EDRF1 controls the differentiation of both division cycle genes, end undifferentiated genes, and initiate differentiation genes $[59,60]$. Thus, EDRF1 may be involved in the proliferation and differentiation of erythrocytes in the blood during hypoxia adaptation in L. crocea. Both RPS16 and RPL8 are core genes of the darkorange module, and both important components of the mature ribosome structure, which are not only involved in protein synthesis, but also have a variety of extra-ribosomal functions, such as regulating cell growth, proliferation, differentiation, apoptosis, and DNA repair [61-63]. This implies that RPS16 and RPL8 may not only be involved in hemoglobin synthesis but also play an important role in the proliferation and differentiation of erythrocytes in a hypoxic environment. In addition, results from the KEGG analysis showed significant enrichment of the VEGF signaling pathway, insulin signaling pathway, mTOR signaling pathway, and apoptosis, indicating that oxygen transport under hypoxia adaptation is a complex process that requires the assistance of signal transduction.

The magenta module contained 494 genes, of which 4 were annotated to hub genes. The GO enrichment analysis of the magenta module showed that potassium ion transmembrane transporter activity, intracellular ligand-gated ion channel activity, and other ion regulatory-related terms were significantly enriched. This implies that the magenta module may be related to ion regulation. KCNK5 which belongs to the G protein-sensitive inwardly rectifying potassium channel family is involved in potassium ion transport in epithelial cells [64]. Correlational studies indicate that KCNK5 plays an important role in apoptotic volume reduction, cell volume regulation, and regulation of negative membrane potential [65]. This implies that it may be involved in several functions such as apoptosis and ion regulation under hypoxia. Furthermore, both SNAT2 and SLC22A7 are transmembrane carriers. SNAT2 is dependent on the extracellular sodium ion concentration, and its best transport substrate is alanine, the most widely distributed transport protein in the family; SLC22A7 is a known cotransporter of sodium and phosphate, and can transport organic anions [66-68]. Moreover, KIF3B is a class of protein macromolecules with cellular motor function and involved in power supply (energy generated by ATP hydrolysis) for intracellular material transport, replication of DNA genetic material, transcription and translation of genetic material, and cell division $[69,70]$. These functions imply that KIF3B may provide transport pathways and power for certain protein complexes in the ion regulation process under hypoxia in L. crocea. In addition, KEGG enrichment analysis also showed that pathways such as the p53 and PPAR signaling pathways were significantly enriched, indicating that the magenta module is associated with central signaling which also affects ion regulatory processes under hypoxia.

The saddlebrown module contained 411 genes, of which 4 hub genes were annotated. Energy metabolism is important for hypoxia adaptation in L. crocea, and the GO enrichment analysis of genes in the saddlebrown module was significantly enriched for functional modules (ATPase activity, steroid hydroxylase activity, carbohydrate phosphorylation, etc.) mainly related to energy metabolism. In the KEGG enrichment analysis, arginine and proline metabolism, glycolysis/gluconeogenesis, and carbon metabolism were significantly enriched, indicating that energy metabolism plays an important role in the hypoxia adaptation of L. crocea. PFKL is a rate-limiting enzyme of glycolysis, which is inhibited by high concentrations of ATP and citric acid and activated by adenosine monophosphate and fructose-2,6-diphosphate. Several recent studies have found that hypoxia-inducible factor $1 \alpha(H I F-1 \alpha)$ in hypoxic environments increases PFKL activity by regulating its gene expression to accelerate the rate of glycolysis and thus meet the energy requirements when 
oxygen supply is insufficient [71,72]. PFKL as one of the hub genes, may be important in regulating the rate of glucose metabolism in L. crocea in a hypoxic environment. GSK-3 $\beta$ is a key enzyme in the glycogen metabolic pathway and plays an important role in the formation of the $\alpha-1,4$ glucosidic bond [73]. However, GSK-3 $\beta$ also plays an important role in the regulation of a variety of cellular functions, including the regulation of cell metabolism and signaling, cell structure and motility, and microtubule motility [74,75]. For example, under hypoxic conditions, suppression of GSK-3 $\beta$ expression down-regulates p70S6K1 protein phosphorylation levels and regulates the expression of its downstream signaling molecules HIF-1 $\alpha$ and VEGF, therefore inhibiting angiogenesis and apoptosis [76]. GSK-3 $\beta$, a hub gene in the saddlebrown module, may be involved in various aspects of the regulation of hypoxia adaptation in L. crocea. PC, an important enzyme in glucose metabolism, is expressed in the liver, pancreas, and adipose tissue and is responsible for regulating gluconeogenesis and fatty acid synthesis [77]. In tumor cells, the expression level of $P C$ is significantly increased to catalyze the generation of more pyruvate to generate oxaloacetic acid to enter the tricarboxylic acid cycle pathway, thus meeting the energy needs of tumor cells for rapid value addition $[78,79]$. The growth of tumor cells has been shown to be significantly inhibited when PC expression is inhibited [80]. PC, one of the hub genes of the module, may be related to the promotion of glycolysis in a hypoxic environment to accelerate oxidative energy supply. AKR1A1 catalyzes the conversion of ethanol from ethylene to hydrogen ions and is present in plants, microorganisms, and most mammals. However, it has been shown that AKR1A1 can convert lactic acid produced by anaerobic metabolism into ethanol in the body and excrete it through the gills under hypoxic conditions in carp such as goldfish, Carassius auratus, and Rhodeus amarus, thus avoiding acidosis due to excessive accumulation of lactic acid [81-83]. As AKR1A1 was one of the core genes in this module, it implies that $A K R 1 A 1$ may have some undiscovered functions or that ethanol metabolic pathways may play a role in L. crocea during hypoxia adaptation (requiring the measurement of ethanol levels in their bodies and environment to be certain). These genes have been studied in more detail in mammals and zebrafish, and less so in L. crocea necessitating more research.

The darkolivegreen module contained 181 genes, of which 3 hub genes were annotated. $A C A C A$ is a key enzyme in lipid metabolism that catalyzes the generation of malonyl coenzyme A from acetyl coenzyme A. It is the first rate-limiting enzyme in fatty acid synthesis and plays an important role in carbohydrate and fat metabolism [84]. It has been shown that a significantly high expression of $A C A C A$ protects cells from apoptosis due to hypoxia and is a key gene in regulating the metabolic state of cells [85]. This implies that $A C A C A$ may regulate key processes such as lipid metabolism during hypoxia in L. crocea. HSP90B1 is a genetically highly conserved member of the heat shock protein family, and its main function is to act as a molecular chaperone involved in the correct post-translational folding processing of proteins, in addition to anti-apoptosis, regulation of the cell cycle, immune regulation, and participation in cell movement, and migration and other functions [86,87]. Studies have shown that HSP90B1 is a major regulator of $H I F-1 \alpha$ activation under hypoxia, suggesting that this gene may be a major regulator of hypoxia stress in L. crocea [86]. PIK3CD, the main downstream molecule of tyrosine kinases and $\mathrm{G}$ protein-coupled receptors, catalyze the production of the second messenger phosphatidylinositol 3,4,5-trisphosphate, and activate numerous downstream activators such as Akt, GSK-3, Forkhead transcription factor FOXO1, and mTOR, thus playing an important role in regulating cell proliferation, differentiation, apoptosis, and glucose transport.

\section{Materials and Methods}

\subsection{Experimental Materials and Hypoxia Experiments}

Large yellow croakers were obtained from the farmed cages in Ningde, Fujian Province, China. The healthy large yellow croakers (weight $63.61 \pm 6.63 \mathrm{~g}$, length $15.90 \pm 1.52 \mathrm{~cm}$ ) were randomly selected from the same batch of farmed cages and transported to an indoor concrete pond for 14 days (DO $7.80 \pm 0.5 \mathrm{mg} / \mathrm{L}, 22 \pm 0.5^{\circ} \mathrm{C}$ ). The fish were fed an artificial 
compound feed containing about $12 \%$ moisture, $40 \%$ protein, $5 \%$ crude fat, $5 \%$ crude fiber and 15\% crude ash (Fujian Yuehai Feed Co. Ltd., China) twice a day (6:00 a.m. and 5:00 p.m.), and the water was cleaned and changed to maintain water quality. The diet was stopped one day prior to starting experimentation.

The hypoxia experiments were carried out in accordance with the laboratory's previously described protocols [9]. L. crocea were placed in hypoxia water (DO $2.0 \pm 0.1 \mathrm{mg} / \mathrm{L}$ ) for $96 \mathrm{~h}$ and then returned to normal DO $(7.80 \pm 0.1 \mathrm{mg} / \mathrm{L})$ for $24 \mathrm{~h}$. Three L. crocea from each of the three parallel tanks were taken at $0,6,24,48,96$, and $120 \mathrm{~h}$. The fish were euthanized with 0.05\% MS-222 (3-Aminobenzoic acid ethyl ester methanesulfonate, Sigma, Saint Louis, MO, USA), and their livers and gills were collected on ice trays, snap-frozen with liquid nitrogen, and stored at $-80^{\circ} \mathrm{C}$. The blood was drawn from the tail vein, stored in liquid nitrogen, and stored at $-80^{\circ} \mathrm{C}$ for later use.

The principles and procedures of the sampling methods were in strict accordance with the requirements of the Governing Regulation for the Use of Experimental Animals in Zhejiang Province (Zhejiang Provincial Government Order No. 263, released in 17 August 2009, effective from 1 October 2010) and approved by the Animal Care and Use Committee of Ningbo University.

\subsection{RNA Sequencing}

Total RNA from blood, gills, and liver tissues was extracted at 0, 6, 24, 48, 96, and $120 \mathrm{~h}$ using TRIzol (equal mix of RNA from three fish) (Invitrogen, Carlsbad, CA, USA) and recorded as either Bl-0h, Bl-6h, Bl-24 h, Bl-48 h, Bl-96 h, Bl-120 h; Gi-0h, Gi-6h, Gi-24 h, Gi-48 h, Gi-96 h, Gi-120 h; or Li-0h, Li-6h, Li-24 h, Li-48 h, Li-96 h, or Li-120 h, respectively. The concentrations of RNA $\left(\mathrm{OD}_{260 / 280}\right.$ and $\left.\mathrm{OD}_{260 / 230}\right)$ of each sample were measured using a NanoPhotometer spectrophotometer (Thermo Fisher Scientific, Waltham, MA, USA). RNA integrity and nucleic acid absorption peaks were detected using an Agilent 2100 Bioanalyzer (Agilent Technologies, Santa Clara, CA, USA). RNA samples with sample concentrations $\geq 200 \mathrm{ng} / \mu \mathrm{L}, \mathrm{OD}_{260 / 280}$ between $1.8-2.2, \mathrm{OD}_{260 / 230} \geq 2.0$, and relative intensity noise (RIN) $\geq 7.0$, were selected for subsequent library construction.

The above qualified sample $(1 \mu \mathrm{g})$ was used for cDNA library construction using the NEBNext ${ }^{\circledR}$ Ultra $^{\mathrm{TM}}$ RNA Library Prep Kit (Illumina, San Diego, CA, USA). After the libraries passed quality control, the libraries of different samples were pooled according to the effective concentration and the target downstream data volume required, and then the 18 cDNA libraries built were sequenced in both directions using the HiSeq 2500 platform (Illumina, USA), with a sequencing read length of $125 \mathrm{bp}$ at one end and a sequencing volume of $6 \mathrm{~Gb}$ per sample.

\subsection{Data Processing and Analysis}

The Fast QC software (version 0.11.8) was used to remove the adapters, unknown bases (ploy-N), and repeat and low-quality fragments from the raw reads obtained by sequencing to obtain high-quality purity data (clean reads) used for subsequent data analysis [88]. The Q20, Q30, and GC content of the clean reads were also counted. The software Hisat2 (version 2.1.0) was used to compare clean reads with the reference genome of L. crocea (GenBank assembly accession: GCA_000972845.2) to obtain information about their position on the reference genome and sequence features unique to the sequenced samples [89]. All the raw data have been uploaded to the NCBI website (accession numbers: SRR11300734-SRR11300741 and SRR11300745-SRR11300754).

Before performing differential expression analysis, the clean reads data of each sample was normalized using the edgeR package (version 1.0.0) [90]. Then, the EBseq R package (version 1.30.0) was used to perform differential expression analysis on every two samples, with false discovery rate $(F D R)<0.05$, and $\mid \log 2$ (fold change) $\mid \geq 1$ as the threshold for significant differential expression [91]. Genes that met these criteria were identified as DEGs. Then, the GOseq R package (version 1.42.0) and KOBAS were used to perform GO and KEGG significant enrichment analyses for DEGs [92,93]. 


\subsection{Weighted Gene Co-Expression Network Analysis}

The WGCNA package (version 1.70-3) in R was used to construct a weighted gene co-expression network and divide related modules according to the steps described by Langfelder and Horvath [20]. To ensure that the resulting network is close to the scalefree topology (linear regression model satisfies $R^{2}=0.8$ ), we first use the function in the WGCNA package to calculate the weighting coefficient $\beta$. Hierarchical clustering was then performed on 18,054 genes in the 18 transcriptome libraries, and the resulting gene tree map was cut by dynamic cutting to obtain gene collections (modules). The minimum number of genes in a module was set to 100, and modules with similar expression patterns were merged according to the similarity of module eigenvalues (0.75), and other parameters were set according to the default settings.

The eigengene value for each module was calculated using all genes within each module, and correlated this value with each sample. Only $p$ values less than 0.05 were considered to be hypoxia-related modules. Genes within the highly related modules were subjected to GO enrichment and KEGG enrichment analyses. Genes with the top $1 \% \mathrm{kME}$ (module membership) values were used as hub genes, and Cytoscape software (version 3.7.2) was used to construct the visualization network [94].

\subsection{Quantitative Real-Time PCR Analysis}

To verify the accuracy of the RNA-seq results, qRT-PCR was performed on a total of eight DEGs (for some tissue samples with non-DEGs) from three tissues of $L$. crocea using the extracted RNA. The RNA to be tested was first reverse transcribed into cDNA using the PrimeScript ${ }^{\mathrm{TM}}$ RT reagent Kit (Takara, Dalian, China) as a template for real-time fluorescent quantitative PCR analysis, followed by quantitative PCR experiments on a Roche Light Cycler 96 using SYBR Green Master I (Roche, Basel, Switzerland). The primers for the target genes were synthesized and provided by BGI Genomics (Shenzhen, China) (see Supplementary Table S4), and the reference gene was $\beta$-actin. qRT-PCR was performed with three biological replicates per group and three technical replicates, and the results were analyzed using the $2^{-\Delta \Delta c t}$ method.

\subsection{Statistical Analysis}

Statistical analyses were performed using SPSS 19.0 (IBM, Armonk, NY, USA) and Microsoft Excel software. Group means were compared using a one-way analysis of variance. $p<0.05$ was considered statistically significant.

\section{Conclusions}

In summary, L. crocea triggers a variety of processes within tissue cells to adapt to hypoxia and reoxygenation environments, including adaptive regulation of energy metabolism, oxygen transport, and ion homeostasis, along with the involvement of various signaling pathways (Figure 8). In addition, some hub genes within the association module were uncovered, such as RPS16, ISCA1, EDRF1, RPL8, KCNK5, SNAT2, KIF3B, SLC22A7, PFKL, GSK-3 $3, P C, A K R 1 A 1, A C A C A, H S P 90 B 1$, and PIK3CD, which could provide clues for further studies on hypoxia adaptation mechanisms in L. crocea. In conclusion, this study provides the first phylogenetic insight into the molecular mechanisms underlying the physiological changes in L. crocea under hypoxia and reoxygenation stress, which can provide an important reference for subsequent studies on the regulatory mechanisms related to hypoxia tolerance in $L$. crocea. 


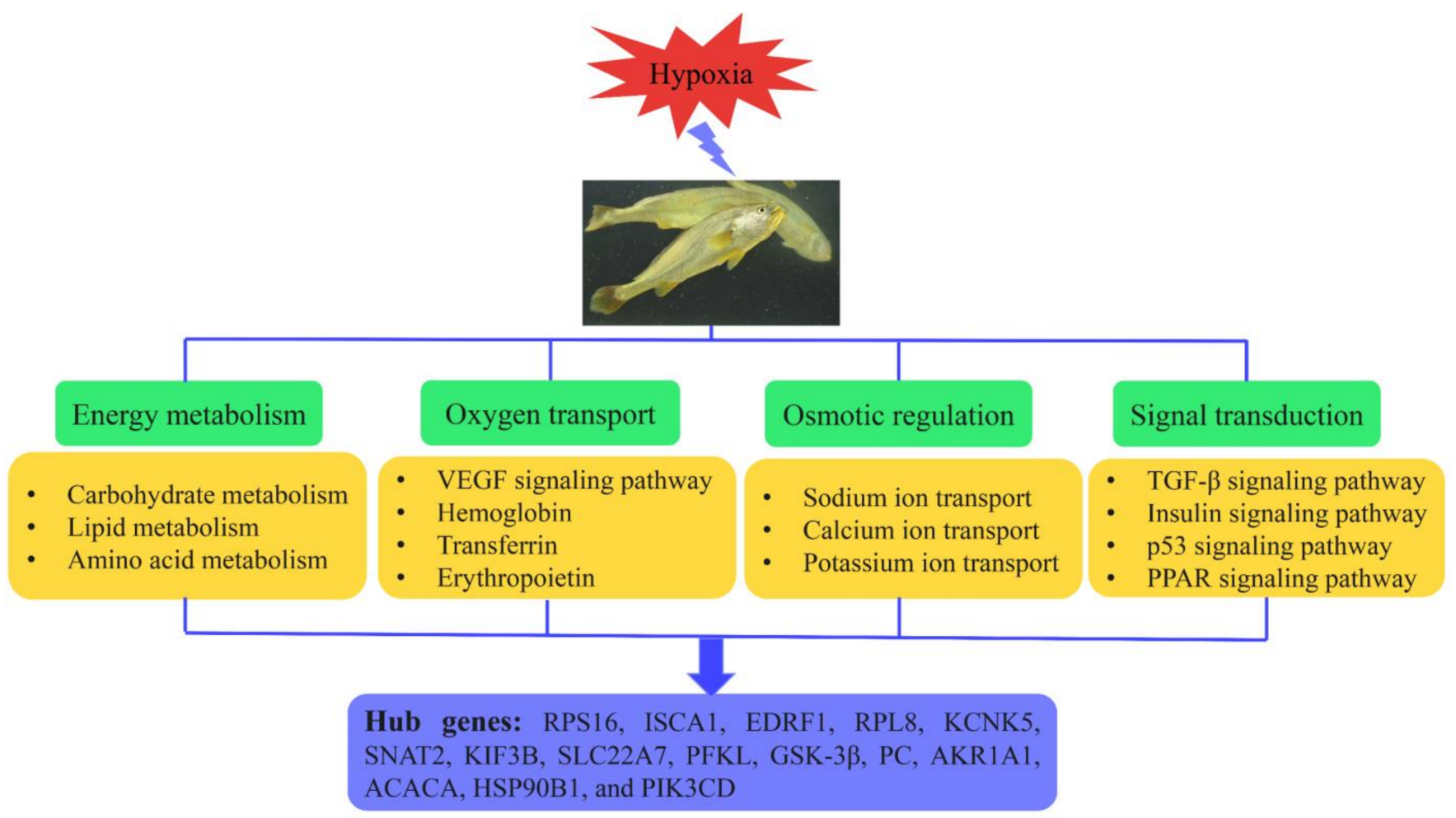

Figure 8. Diagram of hypoxia adaptation strategies and regulatory hub genes in L. crocea. See text for abbreviations and explanations.

Supplementary Materials: The following are available online at https:/ / www.mdpi.com/article/ 10.3390/ani11113021/s1. Figure S1: Top GO enrichment results of DEGs at different time points in the blood (A), gills (B), and liver (C) of L. crocea (The first 5 terms were selected according to KS values), Figure S2: An overview of the KEGG pathways significantly enriched in differentially expressed genes in the blood, Figure S3: An overview of the KEGG pathways significantly enriched in differentially expressed genes in the gills, Figure S4: An overview of the KEGG pathways significantly enriched in differentially expressed genes in the liver, Figure S5: Validation RNA-seq profiles by qRT-PCR. Relative mRNA expression valves $(n=3)$. Data were expressed as mean \pm standard deviation, Table S1: Summary of the Illumina sequencing and mapping statistics of the 18 transcriptome samples, Table S2: Differentially expressed genes under different time and tissues of $L$. crocea (FDR $<0.05$ and $\mid \log 2$ (fold change) $\mid>1$ ), Table S3: Detailed list of gene members of each module analyzed by WCCNA in L. crocea, Table S4: The sequence of qRT-PCR specific primers of differentially expressed genes in L. crocea.

Author Contributions: Conceptualization, Y.Z. and J.D.; Data curation, Y.Z. and S.L.; Formal analysis, Y.Z. and J.D.; Funding acquisition, X.W. (Xiongfei Wu) and J.Z.; Investigation, Y.W., J.W. and W.S.; Methodology, J.D., C.L. and X.W. (Xuelei Wang); Project administration, W.S. and J.Z.; Resources, X.W. (Xuelei Wang) and W.S.; Software, J.D.; Supervision, J.Z.; Validation, Y.Z. and W.S.; Writing-original draft, Y.Z. and J.D.; Writing-review and editing, Y.Z., J.D. and X.G. All authors have read and agreed to the published version of the manuscript.

Funding: This research was funded by the China Agriculture Research System of MOF and MARA (CARS-47), the Fourteenth Five-year Aquatic Breeding Special Project of Zhejiang Province (No. 2021C02069-1), the "Science and Technology Innovation 2025" Major Special Project of Ningbo City (No. 2021Z002), the Scientific and Technical Project of Zhejiang Province (No. 2016C02055-7), the Scientific and Technical Project of Ningbo City (No. 2015C110005), the NSFC-Zhejiang Joint Fund for the Integration of Industrialization and Informatization (No. U1809212), the research on all-male and resistance breeding technology of large yellow croaker, the Collaborative Innovation Center for Zhejiang Marine High-efficiency and Healthy Aquaculture, and the K.C. Wong Magna Fund in Ningbo University. 
Institutional Review Board Statement: The principles and procedures of the sampling methods were in strict accordance with the requirements of the Governing Regulation for the Use of Experimental Animals in Zhejiang Province (Zhejiang Provincial Government Order No. 263, released in 17 August 2009, effective from 1 October 2010) and approved by the Animal Care and Use Committee of Ningbo University.

Data Availability Statement: All the raw data have been uploaded to the NCBI website (accession numbers: PRJNA612352).

Conflicts of Interest: The authors declare no conflict of interest.

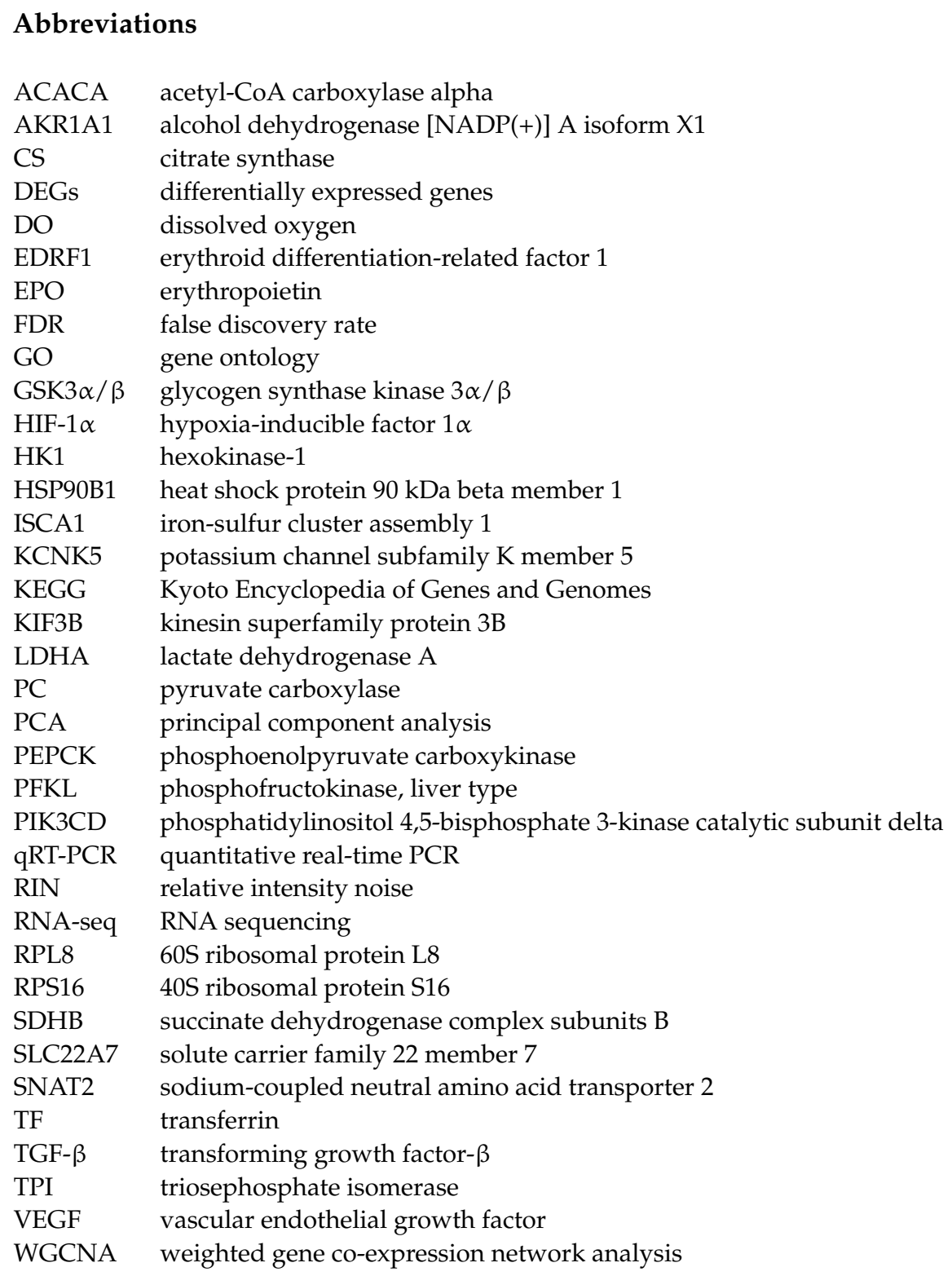

\section{References}

1. Conley, D.J.; Carstensen, J.; Vaquer-Sunyer, R.; Duarte, C.M. Ecosystem thresholds with hypoxia. Hydrobiologia 2009, 629, 21-29. [CrossRef]

2. Hou, Z.S.; Wen, H.S.; Li, J.F.; He, F.; Li, Y.; Qi, X. Environmental hypoxia causes growth retardation, osteoclast differentiation and calcium dyshomeostasis in juvenile rainbow trout (Oncorhynchus mykiss). Sci. Total Environ. 2020, 705, 135272. [CrossRef]

3. Lee, J.; Park, K.-T.; Lim, J.-H.; Yoon, J.-E.; Kim, I.-N. Hypoxia in Korean Coastal Waters: A Case Study of the Natural Jinhae Bay and Artificial Shihwa Bay. Front. Mar. Sci. 2018, 5, 70, 1-19. [CrossRef]

4. Stow, C.A.; Qian, S.S.; Craig, J.K. Declining threshold for hypoxia in the Gulf of Mexico. Environ. Sci. Technol. 2005, 39, 716-723. [CrossRef] [PubMed] 
5. Conley, D.J.; Carstensen, J.; Aigars, J.; Axe, P.; Bonsdorff, E.; Eremina, T.; Haahti, B.-M.; Humborg, C.; Jonsson, P.; Kotta, J.; et al. Hypoxia Is Increasing in the Coastal Zone of the Baltic Sea. Environ. Sci. Technol. 2011, 45, 6777-6783. [CrossRef] [PubMed]

6. Chi, L.; Song, X.; Yuan, Y.; Wang, W.; Cao, X.; Wu, Z.; Yu, Z. Main factors dominating the development, formation and dissipation of hypoxia off the Changjiang Estuary (CE) and its adjacent waters, China. Environ. Pollut. 2020, 265, 115066. [CrossRef]

7. Wu, R.S.S. Hypoxia: From molecular responses to ecosystem responses. Mar. Pollut. Bull. 2002, 45, 35-45. [CrossRef]

8. Ao, J.; Mu, Y.; Xiang, L.X.; Fan, D.; Feng, M.; Zhang, S.; Shi, Q.; Zhu, L.Y.; Li, T.; Ding, Y.; et al. Genome sequencing of the perciform fish Larimichthys crocea provides insights into molecular and genetic mechanisms of stress adaptation. PLoS Genet. 2015, 11, e1005118. [CrossRef]

9. Ding, J.; Liu, C.; Luo, S.; Zhang, Y.; Gao, X.; Wu, X.; Shen, W.; Zhu, J. Transcriptome and physiology analysis identify key metabolic changes in the liver of the large yellow croaker (Larimichthys crocea) in response to acute hypoxia. Ecotoxicol. Environ. Saf. 2020, 189, 109957. [CrossRef]

10. Sun, P.; Bao, P.; Tang, B. Transcriptome analysis and discovery of genes involved in immune pathways in large yellow croaker (Larimichthys crocea) under high stocking density stress. Fish. Shellfish Immunol. 2017, 68, 332-340. [CrossRef] [PubMed]

11. Wang, Q.F.; Shen, W.L.; Hou, C.C.; Liu, C.; Wu, X.F.; Zhu, J.Q. Physiological responses and changes in gene expression in the large yellow croaker Larimichthys crocea following exposure to hypoxia. Chemosphere 2017, 169, 418-427. [CrossRef] [PubMed]

12. Aoki, T.; Hirono, I.; Kondo, H.; Hikima, J.-I.; Jung, T.S. Microarray technology is an effective tool for identifying genes related to the aquacultural improvement of Japanese flounder. Paralichthys Olivaceus. Comp. Biochem. Physiol. D Genom. Proteom. 2011, 6, 39-43. [CrossRef] [PubMed]

13. Douglas, S.E. Microarray studies of gene expression in fish. OMICS 2006, 10, 474-489. [CrossRef] [PubMed]

14. Wenne, R.; Handschuh, L.; Pocwierz-Kotus, A.; Urbaniak, R.; Formanowicz, P.; Calkiewicz, J.; Brzozowska, K.; Figlerowicz, M.; Wegrzyn, G.; Wrobel, B. The application of microarray technology to the identification of Tc1-like element sequences in fish genomes. Mar. Biol. Res. 2011, 7, 466-477. [CrossRef]

15. Xiaolin, L.; Lei, C.; Peng, X.; Guoqing, L.; Michael, W.; Xiaowen, S.; Songlin, C. Transcriptome analysis of crucian carp (Carassius auratus), an important aquaculture and hypoxia-tolerant species. PLoS ONE 2013, 8, e62308.

16. Marques, I.J.; Leito, J.T.D.; Spaink, H.P.; Testerink, J.; Jaspers, R.T.; Witte, F.; Berg, S.V.D.; Bagowski, C.P. Transcriptome analysis of the response to chronic constant hypoxia in zebrafish hearts. J. Comp. Physiol. B Biochem. Syst. Environ. Physiol. 2008, 178, 77-92. [CrossRef] [PubMed]

17. Kim, A.; Yoon, D.; Lim, Y.; Roh, H.J.; Kim, S.; Park, C.-I.; Kim, H.-S.; Cha, H.-J.; Choi, Y.H.; Kim, D.-H. Co-Expression Network Analysis of Spleen Transcriptome in Rock Bream (Oplegnathus fasciatus) Naturally Infected with Rock Bream Iridovirus (RBIV). Int. J. Mol. Sci. 2020, 21, 1707. [CrossRef]

18. Zhao, X.; Yu, H.; Kong, L.; Li, Q. Gene Co-Expression Network Analysis Reveals the Correlation Patterns Among Genes in Euryhaline Adaptation of Crassostrea gigas. Mar. Biotechnol. 2016, 18, 535-544. [CrossRef] [PubMed]

19. Huang, Z.; Ma, A.; Yang, S.; Liu, X.; Zhao, T.; Zhang, J.; Wang, X.-A.; Sun, Z.; Liu, Z.; Xu, R. Transcriptome analysis and weighted gene co-expression network reveals potential genes responses to heat stress in turbot Scophthalmus maximus. Comp. Biochem. Physiol. D Genom. Proteom. 2020, 33, 100632. [CrossRef]

20. Langfelder, P.; Horvath, S. WGCNA: An R package for weighted correlation network analysis. BMC Bioinform. 2008, 9, 559. [CrossRef]

21. Tang, Y.; Xin, G.; Zhao, L.M.; Huang, L.X.; Qin, Y.X.; Su, Y.Q.; Zheng, W.Q.; Wu, B.; Lin, N.; Yan, Q.P. Novel insights into host-pathogen interactions of large yellow croakers (Larimichthys crocea) and pathogenic bacterium Pseudomonas plecoglossicida using time-resolved dual RNA-seq of infected spleens. Zool. Res. 2020, 41, 314-327. [CrossRef]

22. Jian Xu, Q.L.L.X. Shaolin Wang, Yanliang Jiang, Zixia Zhao, Yan Zhang, Jiongtang Li, Chuanju Dong, Peng Xu, Xiaowen Sun, Gene expression changes leading extreme alkaline tolerance in Amur ide (Leuciscus waleckii) inhabiting soda lake. BMC Genom. 2013, 14, 1-11.

23. Guo, L.; Wang, Y.; Liang, S.; Lin, G.; Chen, S.; Yang, G. Tissue-overlapping response of half-smooth tongue sole (Cynoglossus semilaevis) to thermostressing based on transcriptome profiles. Gene 2016, 586, 97-104. [CrossRef]

24. Yang, Y.; Fu, Q.; Wang, X.; Liu, Y.; Zeng, Q.; Li, Y.; Gao, S.; Bao, L.; Liu, S.; Gao, D.; et al. Comparative transcriptome analysis of the swimbladder reveals expression signatures in response to low oxygen stress in channel catfish, Ictalurus punctatus. Physiol. Genom. 2018, 50, 636-647. [CrossRef]

25. Zhang, G.; Yin, S.; Mao, J.; Liang, F.; Zhao, C.; Li, P.; Zhou, G.; Chen, S.; Tang, Z. Integrated analysis of mRNA-seq and miRNA-seq in the liver of Pelteobagrus vachelli in response to hypoxia. Sci. Rep. 2016, 6, 22907. [CrossRef] [PubMed]

26. Kubiczkova, L.; Sedlarikova, L.; Hajek, R.; Sevcikova, S. TGF- $\beta$-An excellent servant but a bad master. J. Transl. Med. 2012, 10, 1-24. [CrossRef] [PubMed]

27. Shi, Y.; Massagué, J. Mechanisms of TGF- $\beta$ Signaling from Cell Membrane to the Nucleus. Cell 2003, 113, 685-700. [CrossRef]

28. Teleman, A.A. Molecular mechanisms of metabolic regulation by insulin in Drosophila. Biochem. J. 2009, 425, 13-26. [CrossRef]

29. Eckel, J.; Reinauer, H. Insulin action on glucose transport in isolated cardiac myocytes: Signalling pathways and diabetes-induced alterations. Biochem. Soc. Trans. 1990, 18, 1125-1127. [CrossRef] [PubMed]

30. Ahren, B. Glucagon-Early breakthroughs and recent discoveries. Peptides 2015, 67, 74-81. [CrossRef]

31. Kular, J.K.; Basu, S.; Sharma, R.I. The extracellular matrix: Structure, composition, age-related differences, tools for analysis and applications for tissue engineering. J. Tissue. Eng. 2014, 5, 1-17. [CrossRef] 
32. Bosman, F.T.; Stamenkovic, I. Functional structure and composition of the extracellular matrix. J. Pathol. 2003, 200, 423-428. [CrossRef] [PubMed]

33. Myllyharju, J.; Schipani, E. Extracellular matrix genes as hypoxia-inducible targets. Cell Tissue Res. 2010, 339, 19-29. [CrossRef] [PubMed]

34. Lin, C.-1.; Zhang, Z.-x.; Tan, Z. Mechanisms of focal adhesion kinase in the proliferation of human pulmonary artery smooth cells under hypoxia. Zhonghua Yi Xue Za Zhi 2011, 91, 2274-2277.

35. Brown, R.C.; Mark, K.S.; Egleton, R.D.; Huber, J.D.; Burroughs, A.R.; Davis, T.P. Protection against hypoxia-induced increase in blood-brain barrier permeability: Role of tight junction proteins and NFkB. J. Cell Sci. 2003, 116, 693-700. [CrossRef] [PubMed]

36. Matey, V.; Richards, J.G.; Wang, Y.; Wood, C.M.; Rogers, J.; Davies, R.; Murray, B.W.; Chen, X.Q.; Du, J.; Brauner, C.J. The effect of hypoxia on gill morphology and ionoregulatory status in the Lake Qinghai scaleless carp, Gymnocypris przewalskii. J. Exp. Biol. 2008, 211, 1063-1074. [CrossRef]

37. Wood, C.M.; Kajimura, M.; Sloman, K.A.; Scott, G.R.; Walsh, P.J.; Almeida-Val, V.M.F.; Val, A.L. Rapid regulation of Na+ fluxes and ammonia excretion in response to acute environmental hypoxia in the Amazonian oscar, Astronotus ocellatus. Am. J. Physiol. Regul. Integr. Comp. Physiol. 2007, 292, R2048-R2058. [CrossRef]

38. Boeuf, G.; Payan, P. How should salinity influence fish growth? Comp. Biochem. Physiol. C Toxicol. Pharmacol. 2001, 130, 411-423. [CrossRef]

39. Marshall, W.S.; Emberley, T.R.; Singer, T.D.; Bryson, S.E.; McCormick, S.D. Time course of salinity adaptation in a strongly euryhaline estuarine teleost, Fundulus heteroclitus: A multivariable approach. J. Exp. Biol. 1999, 202, 1535-1544. [CrossRef]

40. Takahashi, H.; Shibuya, M. The vascular endothelial growth factor (VEGF)/VEGF receptor system and its role under physiological and pathological conditions. Clin. Sci. 2005, 109, 227-241. [CrossRef]

41. Park, K.; Amano, H.; Ito, Y.; Kashiwagi, S.; Yamazaki, Y.; Takeda, A.; Shibuya, M.; Kitasato, H.; Majima, M. Vascular endothelial growth factor receptor-1 (VEGFR-1) signaling enhances angiogenesis in a surgical sponge model. Biomed. Pharmacol. 2016, 78, 140-149. [CrossRef]

42. Maybin, J.A.; Hirani, N.; Brown, P.; Jabbour, H.N.; Critchley, H.O.D. The Regulation of Vascular Endothelial Growth Factor by Hypoxia and Prostaglandin F-2 alpha during Human Endometrial Repair. J. Clin. Endocrinol. Metab. 2011, 96, $2475-2483$. [CrossRef]

43. Li, X.; Li, W.; Feng, S.; Wang, R. Research progress on mechanism in adaptation of hemoglobin to plateau hypoxia. Zhejiang Da Xue Xue Bao. Yi Xue Ban = J. Zhejiang Univ. Med. Sci. 2019, 48, 674-681.

44. Frey, B.J.; Weber, R.E.; van Aardt, W.J.; Fago, A. The haemoglobin system of the mudfish, Labeo capensis: Adaptations to temperature and hypoxia. Comp. Biochem. Physiol. B Biochem. Mol. Biol. 1998, 120, 735-742. [CrossRef]

45. Sun, Y.; Zhu, Z.; Wang, R.; Sun, Y.; Xu, T. Miiuy Croaker Transferrin Gene and Evidence for Positive Selection Events Reveal Different Evolutionary Patterns. PLoS ONE 2012, 7, e43936. [CrossRef] [PubMed]

46. Gomme, P.T.; McCann, K.B. Transferrin: Structure, function and potential therapeutic actions. Drug Discov. Today 2005, 10, 267-273. [CrossRef]

47. Li, Y.R.; Yu, P.; Chang, S.Y.; Wu, Q.; Yu, P.P.; Xie, C.C.; Wu, W.Y.; Zhao, B.L.; Gao, G.F.; Chang, Y.Z. Hypobaric Hypoxia Regulates Brain Iron Homeostasis in Rats. J. Cell. Biochem. 2017, 118, 1596-1605. [CrossRef] [PubMed]

48. Wenger, R.H.; Rolfs, A.; Marti, H.H.; Bauer, C.; Gassmann, M. Hypoxia, a novel inducer of acute-phase gene-expression in a human hepatoma-cell line. J. Biol. Chem. 1995, 270, 27865-27870. [CrossRef] [PubMed]

49. Shah, Y.M.; Xie, L. Hypoxia-Inducible Factors Link Iron Homeostasis and Erythropoiesis. Gastroenterology 2014, 146, 630-642. [CrossRef] [PubMed]

50. Peyssonnaux, C.; Nizet, V.; Johnson, R.S. Role of the hypoxia inducible factors in iron metabolism. Cell Cycle 2008, 7, 28-32. [CrossRef]

51. Zakharov, I.M. Sensitivity of cells to oxygen and erythropoietin production. Rossiiskii Fiziologicheskii Zhurnal Imeni IM Sechenova 2005, 91, 993-1004.

52. Vetrovoy, O.V.; Rybnikova, E.A.; Glushchenko, T.S.; Baranova, K.A.; Samoilov, M.O. Mild hypobaric hypoxic postconditioning increases the expression of HIF-1 alpha and erythropoietin in the CA1 field of the hippocampus of rats that survive after severe hypoxia. Neurochem. J. 2014, 8, 103-108. [CrossRef]

53. Zhao, W.; Langfelder, P.; Fuller, T.; Dong, J.; Li, A.; Hovarth, S. Weighted Gene Coexpression Network Analysis: State of the Art. J. Biopharm. Stat. 2010, 20, 281-300. [CrossRef]

54. Liu, W.; Li, L.; Ye, H.; Tu, W. Weighted gene co-expression network analysis in biomedicine research. Sheng Wu Gong Cheng Xue Bao/Chin. J. Biotechnol. 2017, 33, 1791-1801.

55. Diao, J.; Yu, X.; Wang, X.; Fan, Y.; Wang, S.; Li, L.; Wang, Y.; Xu, L.; Gai, C.; Ye, H.; et al. Full-length transcriptome sequencing combined with RNA-seq analysis revealed the immune response of fat greenling (Hexagrammos otakii) to Vibrio harveyi in early infection. Microb. Pathog. 2020, 149, 104527. [CrossRef] [PubMed]

56. Favaro, E.; Ramachandran, A.; McCormick, R.; Gee, H.; Blancher, C.; Crosby, M.; Devlin, C.; Blick, C.; Buffa, F.; Li, J.-L.; et al. MicroRNA-210 Regulates Mitochondrial Free Radical Response to Hypoxia and Krebs Cycle in Cancer Cells by Targeting Iron Sulfur Cluster Protein ISCU. PLoS ONE 2010, 5, e10345. [CrossRef]

57. Korde, A.S.; Yadav, V.R.; Zheng, Y.-M.; Wang, Y.-X. Primary role of mitochondrial Rieske iron-sulfur protein in hypoxic ROS production in pulmonary artery myocytes. Free Radic. Biol. Med. 2011, 50, 945-952. [CrossRef] 
58. Zheng, Y.M.; Yang, Z.; Joseph, L.; Song, T.; Wang, Y.X. Hypoxia-Induced Ryanodine Receptor-Medicated Ca2+ Release Causes Rieske Iron-Sulfur Protein-Dependent Mitochondrial Reactive Oxygen Species Production in Pulmonary Artery Myocytes. Am. J. Respir. Crit. Care Med. 2015, 191, A5278.

59. Wang, D.C.; Li, Y.; Shen, B.F. Initial function analysis of a novel erythroid differentiation related gene EDRF1. Sci. China Ser. C Life Sci. 2001, 44, 489-496. [CrossRef]

60. Wang, X.; Wang, D.C.; Chen, X.; Hu, M.R.; Wang, J.; Li, Y.; Guo, N.; Shen, B.F. cDNA cloning and function analysis of two novel erythroid differentiation related genes. Sci. China Ser. C Life Sci. 2001, 44, 99-105. [CrossRef]

61. Wood, A.J.; Duff, R.J.; Oliver, M.J. The translational apparatus of Tortula ruralis: Polysomal retention of transcripts encoding the ribosomal proteins RPS14, RPS16 and RPL23 in desiccated and rehydrated gametophytes. J. Exp. Bot. 2000, 51, 1655-1662 [CrossRef] [PubMed]

62. Hou, W.-r.; Hou, Y.-l.; Hao, Y.-z.; Zhang, T.; Peng, Z.-s.; Wu, Y.-S.; Wu, G.-f. cDNA, genomic sequence cloning and overexpression of ribosomal protein S16 gene (RPS16) from the Giant Panda. Afr. J. Biotechnol. 2010, 9, 2185-2191.

63. Chowdhury, R.; Sekirnik, R.; Brissett, N.C.; Krojer, T.; Ho, C.-h.; Ng, S.S.; Clifton, I.J.; Ge, W.; Kershaw, N.J.; Fox, G.C.; et al. Ribosomal oxygenases are structurally conserved from prokaryotes to humans. Nature 2014, 510, 422-426. [CrossRef] [PubMed]

64. Brazier, S.P.; Mason, H.S.; Bateson, A.N.; Kemp, P.J. Cloning of the human TASK-2 (KCNK5) promoter and its regulation by chronic hypoxia. Biochem. Biophys. Res. Commun. 2005, 336, 1251-1258. [CrossRef] [PubMed]

65. Shin, D.H.; Lin, H.; Zheng, H.; Kim, K.S.; Kim, J.Y.; Chun, Y.S.; Park, J.W.; Nam, J.H.; Kim, W.K.; Zhang, Y.H.; et al. HIF-1alphamediated upregulation of TASK-2 $\mathrm{K}(+)$ channels augments $\mathrm{Ca}(2)(+)$ signaling in mouse B cells under hypoxia. J. Immunol. 2014, 193, 4924-4933. [CrossRef] [PubMed]

66. Angelina, R.; Laura, C.B.; Francisco, Z.; Cecilio, G.; Maria Guadalupe, G.-A. Expression of the SNAT2 amino acid transporter during the development of rat cerebral cortex. Int. J. Dev. Neurosci. 2011, 29, 743-748. [CrossRef] [PubMed]

67. Zhang, Z.; Gameiro, A.; Grewer, C. Highly conserved asparagine 82 controls the interaction of Na+ with the sodium-coupled neutral amino acid transporter SNAT2. J. Biol. Chem. 2008, 283, 12284-12292. [CrossRef]

68. Kobayashi, Y.; Ohshiro, N.; Sakai, R.; Ohbayashi, M.; Kohyama, N.; Yamamoto, T. Transport mechanism and substrate specificity of human organic anion transporter 2 (hOat2 SLC22A7). J. Pharm. Pharmacol. 2005, 57, 573-578. [CrossRef]

69. Gumy, L.F.; Chew, D.J.; Tortosa, E.; Katrukha, E.A.; Kapitein, L.C.; Tolkovsky, A.M.; Hoogenraad, C.C.; Fawcett, J.W. The Kinesin-2 Family Member KIF3C Regulates Microtubule Dynamics and Is Required for Axon Growth and Regeneration. J. Neurosci. 2013, 33, 11329-11345. [CrossRef]

70. Sardella, M.; Navone, F.; Rocchi, M.; Rubartelli, A.; Viggiano, L.; Vignali, G.; Consalez, G.G.; Sitia, R.; Cabibbo, A. KIF3C, a novel member of the kinesin superfamily: Sequence, expression, and mapping to human chromosome 2 at 2p23. Genomics 1998, 47, 405-408. [CrossRef]

71. Cota-Ruiz, K.; Leyva-Carrillo, L.; Peregrino-Uriarte, A.B.; Valenzuela-Soto, E.M.; Gollas-Galvan, T.; Gomez-Jimenez, S.; Hernandez, J.; Yepiz-Plascencia, G. Role of HIF-1 on phosphofructokinase and fructose 1, 6-bisphosphatase expression during hypoxia in the white shrimp Litopenaeus vannamei. Comp. Biochem. Physiol. A Mol. Integr. Physiol. 2016, 198, 1-7. [CrossRef]

72. Cota-Ruiz, K.; Peregrino-Uriarte, A.B.; Felix-Portillo, M.; Martinez-Quintana, J.A.; Yepiz-Plascencia, G. Expression of fructose 1,6-bisphosphatase and phosphofructokinase is induced in hepatopancreas of the white shrimp Litopenaeus vannamei by hypoxia. Mar. Environ. Res. 2015, 106, 1-9. [CrossRef]

73. Kaidanovich-Beilin, O.; Woodgett, J.R. GSK-3: Functional insights from cell biology and animal models. Front. Molec. Neurosci. 2011, 4, 1-25. [CrossRef]

74. Ahn, M.; Kim, J.; Park, C.; Cho, J.; Jee, Y.; Jung, K.; Moon, C.; Shin, T. Potential involvement of glycogen synthase kinase (GSK)-3beta in a rat model of multiple sclerosis: Evidenced by lithium treatment. Anat. Cell Biol. 2017, 50, 48-59. [CrossRef] [PubMed]

75. Nagini, S.; Sophia, J.; Mishra, R. Glycogen synthase kinases: Moonlighting proteins with theranostic potential in cancer. Semin. Cancer Biol. 2019, 56, 25-36. [CrossRef] [PubMed]

76. Fluegel, D.; Goerlach, A.; Michiels, C.; Kietzmann, T. Glycogen synthase kinase 3 phosphorylates hypoxia-inducible factor 1 alpha and mediates its destabilization in a VHL-independent manner. Mol. Cell. Biol. 2007, 27, 3253-3265. [CrossRef] [PubMed]

77. Wallace, J.C. My Favorite Pyruvate Carboxylase Feature. IUBMB Life 2010, 62, 535-538. [CrossRef]

78. Lin, Q.; He, Y.; Wang, X.; Zhang, Y.; Hu, M.; Guo, W.; He, Y.; Zhang, T.; Lai, L.; Sun, Z.; et al. Targeting Pyruvate Carboxylase by a Small Molecule Suppresses Breast Cancer Progression. Adv. Sci. 2020, 7, 1903483. [CrossRef] [PubMed]

79. Phannasil, P.; Thuwajit, C.; Warnnissorn, M.; Wallace, J.C.; MacDonald, M.J.; Jitrapakdee, S. Pyruvate Carboxylase Is Up-Regulated in Breast Cancer and Essential to Support Growth and Invasion of MDA-MB-231 Cells. PLoS ONE 2015, 10, e0129848. [CrossRef]

80. Shang, H.; Zheng, J.; Tong, J. Integrated analysis of transcriptomic and metabolomic data demonstrates the significant role of pyruvate carboxylase in the progression of ovarian cancer. Aging 2020, 12, 21874-21889. [CrossRef]

81. Holopainen, I.J.; Tonn, W.M.; Paszkowski, C.A. Tales of two fish: The dichotomous biology of crucian carp (Carassius carassius (L)) in northern Europe. Ann. Zool. Fenn. 1997, 34, 1-22.

82. Shoubridge, E.A.; Hochachka, P.W. Ethanol: Novel end product of vertebrate anaerobic metabolism. Science 1980, 209, 308-309. [CrossRef]

83. Johnston, I.A.; Bernard, L.M. Utilization of the ethanol pathway in carp following exposure to anoxia. J. Exp. Biol. 1983, 104, 73-78. [CrossRef] 
84. Tong, L. Acetyl-coenzyme A carboxylase: Crucial metabolic enzyme and attractive target for drug discovery. Cell. Mol. Life Sci. 2005, 62, 1784-1803. [CrossRef]

85. Keenan, M.M.; Liu, B.; Tang, X.; Wu, J.; Cyr, D.; Stevens, R.D.; Ilkayeva, O.; Huang, Z.; Tollini, L.A.; Murphy, S.K.; et al. ACLY and ACC1 Regulate Hypoxia-Induced Apoptosis by Modulating ETV4 via alpha-ketoglutarate. PLoS Genet. 2015, 11, e1005599. [CrossRef]

86. Zhai, R.; Gong, X.-Y.; Xiong, X.-C.; Jiang, Y.; Huang, Z.-J.; Fang, X. Progress in Molecular Chaperone Regulation of Heat Shock Protein 90 and Cancer. Chin. J. Anal. Chem. 2018, 46, 301-310.

87. Prodromou, C.; Pearl, L.H. Structure and Functional Relationships of Hsp90. Curr. Cancer Drug Targets 2003, 3, 301-323. [CrossRef]

88. Chen, S.; Zhou, Y.; Chen, Y.; Gu, J. fastp: An ultra-fast all-in-one FASTQ preprocessor. Bioinformatics 2018, 34, 884-890. [CrossRef] [PubMed]

89. Daehwan, K.; Ben, L.; Salzberg, S.L. HISAT: A fast spliced aligner with low memory requirements. Nat. Methods 2015, 12, 357-360.

90. Robinson, M.D.; McCarthy, D.J.; Smyth, G.K. edgeR: A Bioconductor package for differential expression analysis of digital gene expression data. Bioinformatics 2010, 26, 139-140. [CrossRef]

91. Leng, N.; Dawson, J.A.; Thomson, J.A.; Ruotti, V.; Rissman, A.I.; Smits, B.M.G.; Haag, J.D.; Gould, M.N.; Stewart, R.M.; Kendziorski, C. EBSeq: An empirical Bayes hierarchical model for inference in RNA-seq experiments. Bioinformatics 2013, 29, 1035-1043. [CrossRef] [PubMed]

92. Young, M.D.; Wakefield, M.J.; Smyth, G.K.; Oshlack, A. Gene ontology analysis for RNA-seq: Accounting for selection bias. Genome Biol. 2010, 11(R14), 1-12. [CrossRef] [PubMed]

93. Mao, X.; Tao, C.J.G.O.; Wei, L. Automated genome annotation and pathway identification using the KEGG Orthology (KO) as a controlled vocabulary. Bioinformatics 2005, 21, 3787-3793. [CrossRef]

94. Shannon, P.; Markiel, A.; Ozier, O.; Baliga, N.S.; Wang, J.T.; Ramage, D.; Amin, N.; Schwikowski, B.; Ideker, T. Cytoscape: A software environment for integrated models of biomolecular interaction networks. Genome Res. 2003, 13, 2498-2504. [CrossRef] [PubMed] 